\title{
20. DEEP SEA DRILLING PROJECT, LEG 3 FORAMINIFERA FROM SELECTED SAMPLES
}

W. H. Blow, British Petroleum Research Centre, London, England

BIOSTRATIGRAPHIC SUMMARY OF AVAILABLE SAMPLES

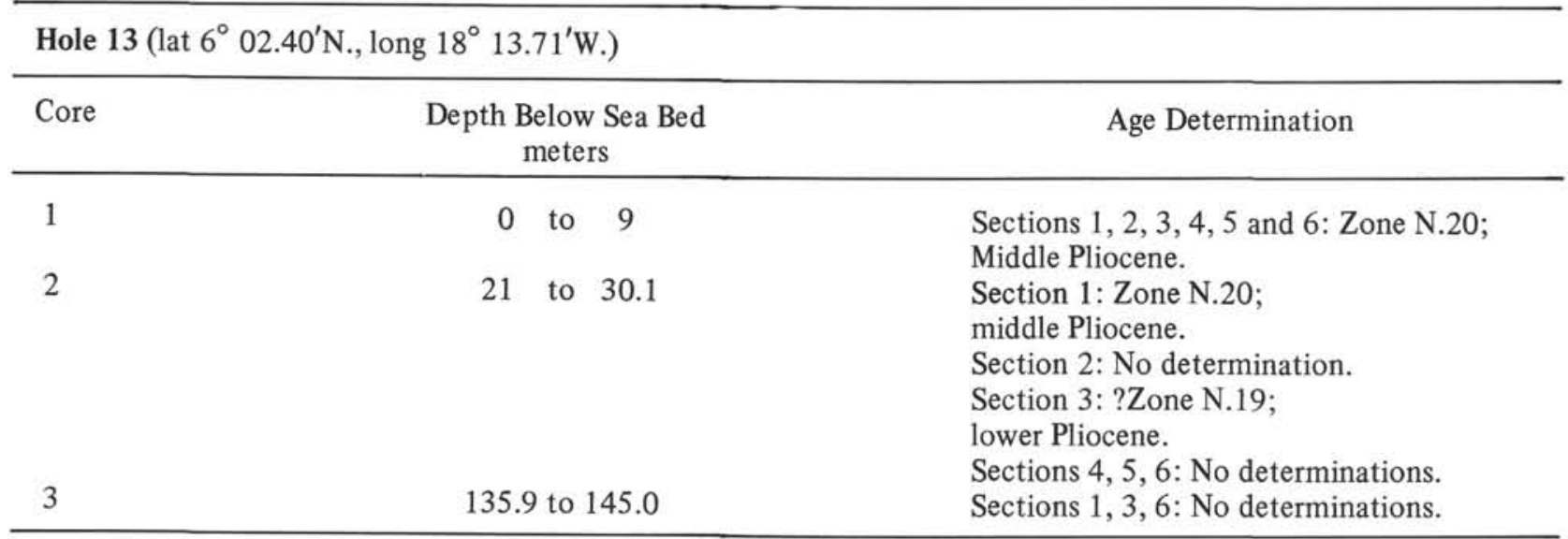

Hole 13A (lat $6^{\circ} 02.40^{\prime} \mathrm{N}$., long $18^{\circ} 13.71^{\prime} \mathrm{W}$.)

\begin{tabular}{ccc} 
Core & Depth Below Sea Bed & Age Determination \\
\hline & meters & Section 1: No determination. \\
1 & 177.3 to 178.9 & Section 1: No determination.
\end{tabular}

Note: In the work recorded here for samples from Leg 3, only the most important and biostratigraphically significant components of the faunas have been noted. No attempt has been made to give an exhaustive faunal analysis of the samples seen. The zonal determinations given for Zones P.13 to N.23 follow the definitions given by Blow (1969). It should be noted that the "P" nomenclature for zones prior to Zone P.13, i.e., Zones P.1 to P.12 inclusive do not follow the system published by Berggren recently in the scheme he attributed to "Blow and Berggren unpublished." In the scheme followed in this work (and which will be published by W. H. Blow) the relationship of the zones to epochs is as shown opposite.

As in Chapter 14, Volume 2, this summary only gives the interpretation of the successions as based on the actual samples investigated. The work does not include data reported by others on a more complete coverage of core-samples from the various holes.
Zone P.15

Zone P.14

Zone P.13

Zone P.12

Zone P.11

Zone P.10

Zone P.9

Zone P.8

Zone P.7

Upper Eocene (part)

Zone P.6

Zone P.5

Zone P.4

Zone P.3

Zone P.2

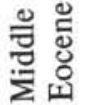

Zone P.1

M. zones.

Cretaceous 
Hole 13A (lat $6^{\circ} 02.40^{\prime} \mathrm{N}$., long $18^{\circ} 13.71^{\prime} \mathrm{W}$.) (Continued)

\begin{tabular}{lcl}
\hline Core & $\begin{array}{c}\text { Depth Below Sea Bed } \\
\text { meters }\end{array}$ & \multicolumn{1}{c}{ Age Determination } \\
\hline 3 & 306.6 to 315.7 & $\begin{array}{l}\text { Sections 1, 2, 3: No determination. } \\
\text { Section 4: }\end{array}$ \\
& & Upper Cretaceous. \\
4 & 379.7 to 381.3 & Section 1: \\
?Cretaceous.
\end{tabular}

Hole 14 (lat $28^{\circ} 19.89^{\prime}$ S., long $20^{\circ} 56.46^{\prime} \mathrm{W}$.)

\begin{tabular}{|c|c|c|c|}
\hline Core & $\begin{array}{r}\text { Depth Below } \\
\text { meters }\end{array}$ & $\checkmark$ Sea Bed & Age Determination \\
\hline $1 \mathrm{~A}$ & 0 to & 9.1 & $\begin{array}{l}\text { Sections 1, 2: ?Zone N.6; } \\
\text { early Miocene. } \\
\text { Section 3: Zone N.5 or Zone N.6; } \\
\text { early Miocene. } \\
\text { Section 4: No determination. } \\
\text { Section 5: Zone N.5 (upper part); } \\
\text { early Miocene. } \\
\text { Section 6: Zone N.5; } \\
\text { early Miocene. }\end{array}$ \\
\hline 2 & 12.2 to & 21.3 & $\begin{array}{l}\text { Section } 1,2,3,4,5 \text { : Zone N.4; } \\
\text { early Miocene. }\end{array}$ \\
\hline 3 & 32.9 to & 42.1 & $\begin{array}{l}\text { Section 1, 2, 3, 4, 5, 6: Zone N.3; } \\
\text { upper Oligocene. }\end{array}$ \\
\hline 4 & 52.4 to & 61.6 & $\begin{array}{l}\text { Sections 1, 2, 3: Zone N.2; } \\
\text { upper Oligocene. }\end{array}$ \\
\hline 5 & 61.6 to & 70.7 & $\begin{array}{l}\text { Sections 1, 2, 3, 4, 5, 6: Zone N.1 (=P.20); } \\
\text { Oligocene. }\end{array}$ \\
\hline 6 & 70.7 to & 79.9 & $\begin{array}{l}\text { Sections } 1,2,3,4,5,6 \text { : } \\
\text { within the interval of } \\
{\left[\begin{array}{l}\text { Zone N.1 (=P.20) early part } \\
\text { Zone P.19, later part }\end{array}\right] \text {; }} \\
\text { Oligocene. }\end{array}$ \\
\hline 7 & 79.9 to & 89.0 & $\begin{array}{l}\text { Sections 1, 2, 3, 4: Zone P.19; } \\
\text { Oligocene. } \\
\text { Sections 5, 6: Zone P.18; } \\
\text { early Oligocene. }\end{array}$ \\
\hline 8 & 89.0 to & 98.2 & $\begin{array}{l}\text { Sections 1, 2, 3, 5, 6: Zone P.18; } \\
\text { early Oligocene. }\end{array}$ \\
\hline 9 & 98.2 to 1 & 106.7 & $\begin{array}{l}\text { Section 2: ?Zone P.18; } \\
\text { early Oligocene. } \\
\text { Sections 3, 4: early Zone P.18 or latest } \\
\text { Zone P.17; } \\
\text { basal Oligocene or latest Eocene. } \\
\text { Sections 5, 6: Zone P.17; } \\
\text { latest Eocene. }\end{array}$ \\
\hline
\end{tabular}




\begin{tabular}{|c|c|c|c|}
\hline \multicolumn{4}{|c|}{ Hole 15 (lat $30^{\circ} 53.38^{\prime} \mathrm{S}$. , long $17^{\circ} 58.99^{\prime} \mathrm{W}$.) } \\
\hline Core & $\begin{array}{r}\text { Depth Below } \\
\text { meters }\end{array}$ & Sea Bed & Age Determination \\
\hline 1 & 0 to & 9.1 & $\begin{array}{l}\text { Sections } 1,2,3,4,5,6 \text { : Zone N.22; } \\
\text { Pleistocene. }\end{array}$ \\
\hline 2 & 13.3 to & 27.4 & $\begin{array}{l}\text { Section 1: basal Zone N.22; } \\
\text { earliest Pleistocene. } \\
\text { Sections 2, 3, 4: ?Zone N.21; } \\
\text { latest Pliocene. } \\
\text { Sections 5, 6; within } \\
{\left[\begin{array}{l}\text { Zone N.21 } \\
\text { Zone N.20 }\end{array}\right] \text { interval; }} \\
\text { middle to late Pliocene. }\end{array}$ \\
\hline 3 & 36.6 to & 45.7 & $\begin{array}{l}\text { Sections 1, 2, 3, 4: Zone N.19; } \\
\text { Pliocene. } \\
\text { Sections 5, 6: upper Zone N.18; } \\
\text { earlier Pliocene. }\end{array}$ \\
\hline 4 & 46.3 to & 55.5 & $\begin{array}{l}\text { Sections 1, 2, 3, 4, 5, 6: Zone N.18; } \\
\text { earlier Pliocene and/or latest Miocene. }\end{array}$ \\
\hline 5 & 77.1 to & 86.3 & $\begin{array}{l}\text { Sections 1, 2, 3, 4, 5, 6: Probably Zone N.17; } \\
\text { later Miocene. }\end{array}$ \\
\hline 6 & 104.8 to & 114.0 & $\begin{array}{l}\text { Sections } 1,2,3,4 \text { : within } \\
{\left[\begin{array}{l}\text { Zone N.17 } \\
\text { Zone N.14 }\end{array}\right] \text { interval; }} \\
\text { middle to late Miocene. } \\
\text { Sections 5, 6: Probably Zone N.15; } \\
\text { middle Miocene. }\end{array}$ \\
\hline 7 & 113.9 to & 123.1 & $\begin{array}{l}\text { Sections 1, 2, 3, 4, 5, 6: Probably Zone N.15; } \\
\text { middle Miocene. }\end{array}$ \\
\hline 8 & 123.1 to & 132.3 & $\begin{array}{l}\text { Sections } 1,2,3,4,5,6: \text { Zone N.6 (lower } \\
\text { part) or ?Zone N.5 (upper part); } \\
\text { Early Miocene. }\end{array}$ \\
\hline 9 & 132.3 to & 140.5 & $\begin{array}{l}\text { Sections } 1,2,3 \text { : Zone N.7; } \\
\text { Early Miocene. } \\
\text { Sections 4, 5, 6: Zone N.6; } \\
\text { Early Miocene (see note in text). }\end{array}$ \\
\hline
\end{tabular}

Hole 16 (lat $30^{\circ} 20.15^{\prime}$ S., long $15^{\circ} 42.79^{\prime} \mathrm{W}$.)

\begin{tabular}{lll}
\hline Core & $\begin{array}{c}\text { Depth Below Sea Bed } \\
\text { meters }\end{array}$ & \multicolumn{1}{c}{ Age Determination } \\
\hline 1 & 0 to 9.2 & $\begin{array}{l}\text { Sections 1, 2: Zone N.23; } \\
\text { Late Pleistocene/Recent. } \\
\text { Section 4: Zone N.22; } \\
\end{array}$ \\
& & Pleistocene. \\
\hline
\end{tabular}


Hole 16 (lat $30^{\circ} 20.15^{\prime}$ S., long $15^{\circ} 42.79^{\prime}$ W.) (Continued)

\begin{tabular}{|c|c|c|}
\hline Core & $\begin{array}{l}\text { Depth Below Sea Bed } \\
\text { meters }\end{array}$ & Age Determination \\
\hline 2 & 18.6 to 27.8 & $\begin{array}{l}\text { Sections } 1,2,3,4,5 \text { : Zone N.22; } \\
\text { Pleistocene. } \\
\text { Section 6: Zone N.21; } \\
\text { late Pliocene. }\end{array}$ \\
\hline 3 & 36.6 to 45.8 & $\begin{array}{l}\text { Sections } 1,2,3,4,5 \text { : Zone N.21; } \\
\text { late Pliocene. } \\
\text { Section 6: within } \\
{\left[\begin{array}{l}\text { Zone N.21 } \\
\text { Zone N.20 }\end{array}\right] \text { interval; }} \\
\text { Pliocene. }\end{array}$ \\
\hline 4 & 54.9 to 64.1 & $\begin{array}{l}\text { Sections 1, 2, 3, 4, 5: within } \\
{\left[\begin{array}{l}\text { Zone N.21 } \\
\text { Zone N.20 }\end{array}\right] \text { interval; }} \\
\text { Pliocene. } \\
\text { Section 6: ?Zone N.19; } \\
\text { Pliocene. }\end{array}$ \\
\hline 5 & 85.7 to 94.9 & $\begin{array}{l}\text { Section 1: ?Zone N.19; } \\
\text { Pliocene. } \\
\text { Section 2: No determination. } \\
\text { Section 3: ?Zone N.19; } \\
\text { Pliocene. } \\
\text { Sections 4, 5, 6: within the } \\
\text { Zone N.19 } \\
\text { Zone N.18 } \\
\text { Pliocene. }\end{array}$ \\
\hline 6 & 104.0 to 113.2 & $\begin{array}{l}\text { Sections 1, 2, 3, 4, 5, 6: Probably Zone } \\
\text { N.18; } \\
\text { earliest Pliocene or latest Miocene. }\end{array}$ \\
\hline 7 & 113.2 to 122.3 & $\begin{array}{l}\text { Sections 1, 2, 3, 4, 5, 6: Probably Zone } \\
\text { N.18; } \\
\text { earliest Pliocene or latest Miocene. }\end{array}$ \\
\hline 8 & 125.4 to 134.5 & $\begin{array}{l}\text { Section 1: Probably Zone N.18; } \\
\text { earliest Pliocene or latest Miocene. } \\
\text { Sections 2, 3, 4, 5, 6: } \\
\text { within }\left[\begin{array}{l}\text { Zone N.18 } \\
\text { Zone N.17 }\end{array}\right] \text { interval; } \\
\text { Late Miocene. }\end{array}$ \\
\hline 9 & 134.5 to 143.7 & $\begin{array}{l}\text { Sections 1, 2, 3, 4: within } \\
{\left[\begin{array}{l}\text { Zone N.18 } \\
\text { Zone N.19 }\end{array}\right] \text { interval; }} \\
\text { Late Miocene. } \\
\text { Section 6: Probably Zone N.17; } \\
\text { Late Miocene. }\end{array}$ \\
\hline
\end{tabular}


Hole 16 (lat $30^{\circ} 20.15^{\prime} \mathrm{S}$., long $15^{\circ} 42.79^{\prime} \mathrm{W}$.) (Continued)

\begin{tabular}{|c|c|c|}
\hline Core & $\begin{array}{c}\text { Depth Below Sea Bed } \\
\text { meters }\end{array}$ & Age Determination \\
\hline 10 & 143.7 to 152.8 & $\begin{array}{l}\text { Section 1: Indeterminate. } \\
\text { Section 2: Probably Zone N.17; } \\
\text { Late Miocene. } \\
\text { Sections 4, 5, 6: Indeterminate. }\end{array}$ \\
\hline 11 & 152.8 to 162.0 & $\begin{array}{l}\text { Section 1: within } \\
{\left[\begin{array}{l}\text { Zone N.17 } \\
\text { Zone N.16 }\end{array}\right] \text { interval; }} \\
\text { Late Miocene. } \\
\text { Sections 2, 3, 4: Indeterminate. } \\
\text { Section 5: Probably Zone N.16; } \\
\text { Late Miocene. } \\
\text { Section 6: within } \\
{\left[\begin{array}{l}\text { Zone N.26 } \\
\text { Zone N.15 upper part }\end{array}\right] ;} \\
\text { Later Miocene. }\end{array}$ \\
\hline
\end{tabular}

Hole 17 (lat $28^{\circ} 02.74^{\prime}$ S., long $6^{\circ} 36.15^{\prime} \mathrm{W}$.)

\begin{tabular}{lll}
\hline Core & $\begin{array}{c}\text { Depth Below Sea Bed } \\
\text { meters }\end{array}$ & \multicolumn{1}{c}{ Age Determination } \\
\hline 1 & 0 to 9.1 & $\begin{array}{l}\text { Sections 1, 2, 3, 4, 5: Zone N.22/Zone N.23; } \\
\text { Pleistocene/Holocene. }\end{array}$ \\
2 & 19.2 to 28.3 & $\begin{array}{l}\text { Sections 1, 2, 3, 4, 5: Zone N.18 or upper- } \\
\text { most Zone N.17; } \\
\text { Late Miocene. } \\
\text { Section 6: Probably Zone N.17; } \\
\text { upper part, Late Miocene. }\end{array}$ \\
\hline
\end{tabular}

Hole 17A (lat $28^{\circ} 02.74^{\prime}$ S., long $6^{\circ} 36.15^{\prime} \mathrm{W}$.)

\begin{tabular}{lll}
\hline Core & $\begin{array}{c}\text { Depth Below Sea Bed } \\
\text { meters }\end{array}$ & \multicolumn{1}{c}{ Age Determination } \\
\hline 1 & 28.3 to 37.5 & Sections 1, 2, 3, 4: Zone N.7; \\
& early Miocene. \\
& Section 5: Probably Zone N.6; \\
& Early Miocene. \\
& Section 6: Probably Zone N.6 or late \\
& Zone N.5; \\
& Early Miocene. \\
& Sections 1, 2, 3: Zone N.5; \\
& Early Miocene. \\
& Sections 4, 5: Zone N.4; \\
& Early Miocene. \\
& Section 6: Probably Zone N.3 upper part or \\
& ?Zone N.4; \\
& earliest Miocene or latest Oligocene. \\
& \\
&
\end{tabular}




\begin{tabular}{|c|c|c|}
\hline \multicolumn{3}{|c|}{ Hole $17 \mathrm{~A}$ (lat $28^{\circ} 02.74^{\prime} \mathrm{S}$. , long $6^{\circ} 36.15^{\prime} \mathrm{W}$.) (Continued) } \\
\hline Core & $\begin{array}{l}\text { Depth Below Sea Bed } \\
\text { meters }\end{array}$ & Age Determination \\
\hline 3 & 85.3 to 94.5 & $\begin{array}{l}\text { Section 1: Zone N.2; } \\
\text { upper Oligocene. } \\
\text { Section 6: Probably upper Zone N.1 } \\
\text { (=P.20); } \\
\text { Oligocene. }\end{array}$ \\
\hline 4 & 94.5 to 102.5 & $\begin{array}{l}\text { Sections 2, 5, 6: Probably Zone P.19; } \\
\text { Oligocene. }\end{array}$ \\
\hline \multicolumn{3}{|c|}{ Hole 17B (lat $28^{\circ} 02.74^{\prime}$ S., long $6^{\circ} 36.15^{\prime}$ W.) } \\
\hline Core & $\begin{array}{l}\text { Depth Below Sea Bed } \\
\text { meters }\end{array}$ & Age Determination \\
\hline 1 & 86.6 to 95.7 & $\begin{array}{l}\text { Sections 1, 2, 3, 4: Zone N.3; } \\
\text { Oligocene. } \\
\text { Sections 5, 6: Zone N.3 or Zone N.2; } \\
\text { Oligocene. }\end{array}$ \\
\hline 2 & 95.7 to 104.8 & $\begin{array}{l}\text { Sections } 1,2,3,4,5: \text { Zone N.2; } \\
\text { Oligocene. } \\
\text { Section 6: early part Zone N.2 or later } \\
\text { part Zone N.1 (=P.20); } \\
\text { Oligocene. }\end{array}$ \\
\hline 3 & 104.9 to 114.0 & $\begin{array}{l}\text { Sections 1, 2, 3, 4, 5, 6: Zone N.1 (=P.20); } \\
\text { Oligocene. }\end{array}$ \\
\hline 4 & 114.0 to 123.2 & $\begin{array}{l}\text { Sections 1, 2: Zone N.1 (=P.20); } \\
\text { Oligocene. } \\
\text { Sections 3, 4, 5, 6: Zone P.19; } \\
\text { Oligocene. }\end{array}$ \\
\hline \multicolumn{3}{|c|}{ Hole 18 (lat $27^{\circ} 58.72^{\prime}$ S., long $08^{\circ} 70^{\prime} \mathrm{W}$.) } \\
\hline Core & $\begin{array}{l}\text { Depth Below Sea Bed } \\
\text { meters }\end{array}$ & Age Determination \\
\hline 1 & 0 to 7.6 & $\begin{array}{l}\text { Section 5: Zone N.22; } \\
\text { Pleistocene. }\end{array}$ \\
\hline 2 & 121.0 to 130.1 & $\begin{array}{l}\text { Sections 1, 2, 3: Zone N.6; } \\
\text { Early Miocene. } \\
\text { Sections 4, 5, 6: Zone N.5; } \\
\text { Early Miocene. }\end{array}$ \\
\hline 3 & 141.1 to 150.3 & $\begin{array}{l}\text { Sections } 1,2,3,4,5 \text { : Zone N.5; } \\
\text { Early Miocene. } \\
\text { Section 6: either lower Zone N.5 or upper } \\
\text { Zone N.4; } \\
\text { Early Miocene. }\end{array}$ \\
\hline
\end{tabular}


Hole 18 (lat $27^{\circ} 58.72^{\prime} \mathrm{S}$., long $08^{\circ} 70^{\prime} \mathrm{W}$.) (Continued)

\begin{tabular}{lll}
\hline Core & $\begin{array}{c}\text { Depth Below Sea Bed } \\
\text { meters }\end{array}$ & \multicolumn{1}{c}{ Age Determination } \\
\hline 4 & 150.3 to 159.4 & $\begin{array}{l}\text { Sections 2, 3, 4, 5: Zone N.4; } \\
\text { Early Miocene. }\end{array}$ \\
5 & 159.7 to 168.8 & $\begin{array}{l}\text { Sections 1, 2, 3, 6: Zone N.4; } \\
\text { Early Miocene. }\end{array}$ \\
6 & 168.8 to 178.0 & $\begin{array}{l}\text { Sections 1, 2, 3, 4, 6: Zone N.3; } \\
\text { Late Oligocene. }\end{array}$ \\
\hline
\end{tabular}

Hole 19 (lat $28^{\circ} 32.08^{\prime} \mathrm{S}$., long $23^{\circ} 40.63^{\prime} \mathrm{W}$.)

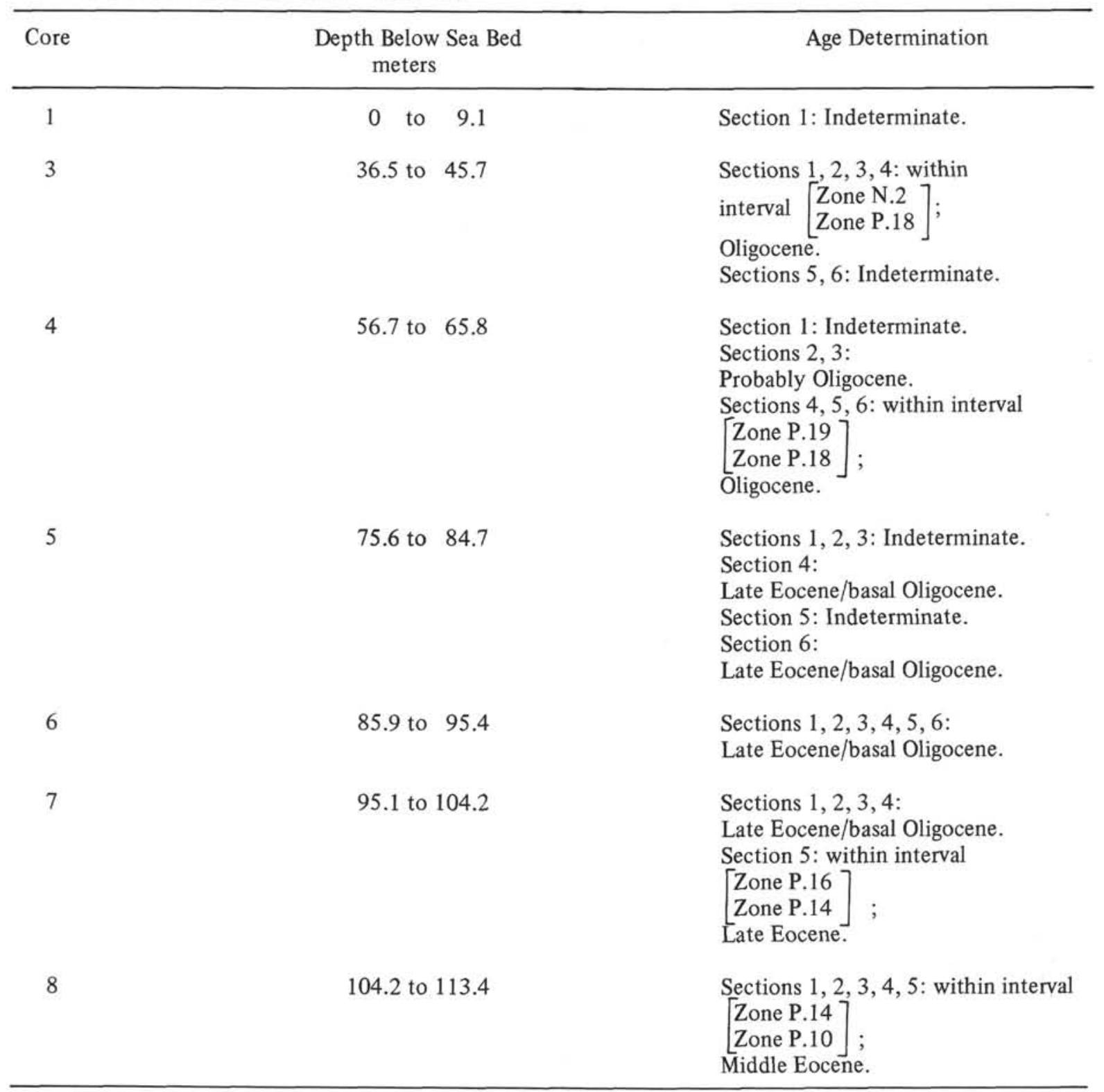




\begin{tabular}{|c|c|c|}
\hline \multicolumn{3}{|c|}{ Hole 19 (lat $28^{\circ} 32.08^{\prime} \mathrm{S}$., long $23^{\circ} 40.63^{\prime} \mathrm{W}$.) (Continued) } \\
\hline Core & $\begin{array}{l}\text { Depth Below Sea Bed } \\
\text { meters }\end{array}$ & Age Determination \\
\hline 8 (Cont) & & $\begin{array}{l}\text { Section 6: within interval } \\
{\left[\begin{array}{l}\text { Zone P.12 } \\
\text { Zone P.11 }\end{array}\right]} \\
\text { lower part Middle Eocene. }\end{array}$ \\
\hline 9 & 113.4 to 122.5 & 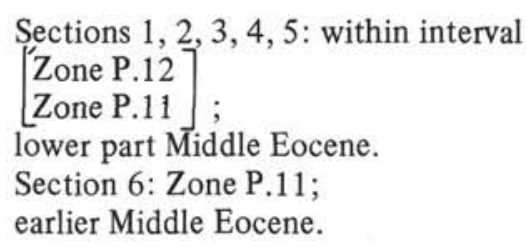 \\
\hline 10 & 123.7 to 132.9 & $\begin{array}{l}\text { Sections 1, 2, 3, 5, 6: Zone P.11; } \\
\text { earlier Middle Eocene. }\end{array}$ \\
\hline 11 & 132.9 to 140.8 & $\begin{array}{l}\text { Sections 1, 2, 3, 4: Zone P.11; } \\
\text { earlier Middle Eocene. }\end{array}$ \\
\hline
\end{tabular}

\begin{tabular}{|c|c|c|}
\hline \multicolumn{3}{|c|}{ Hole 20A (lat $28^{\circ} 31.47^{\prime} \mathrm{S}$., long $26^{\circ} 50.73^{\prime} \mathrm{W}$.) } \\
\hline Core & $\begin{array}{c}\text { Depth Below Sea Bed } \\
\text { meters }\end{array}$ & Age Determination \\
\hline 2 & 15.5 to 24.7 & 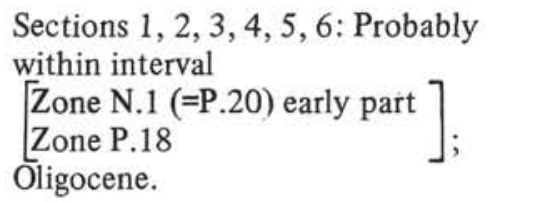 \\
\hline \multicolumn{3}{|c|}{ Hole 20B (lat $28^{\circ} 31.47^{\prime}$ S., long $26^{\circ} 50.73^{\prime} \mathrm{W}$.) } \\
\hline Core & $\begin{array}{l}\text { Depth Below Sea Bed } \\
\text { meters }\end{array}$ & Age Determination \\
\hline 1 & 6.4 to 15.5 & $\begin{array}{l}\text { Sections } 1,2,3,4,5,6 \text { : within interval } \\
{\left[\begin{array}{l}\text { Zone N.1 (=P.20) early part } \\
\text { Zone P.18 }\end{array}\right] \text {; }} \\
\text { Oligocene. }\end{array}$ \\
\hline
\end{tabular}

Hole $20 \mathrm{C}$ (lat $28^{\circ} 31.47^{\prime} \mathrm{S}$., long $26^{\circ} 50.73^{\prime} \mathrm{W}$.)

\begin{tabular}{cccc}
\hline Core & $\begin{array}{c}\text { Depth Below Sea Bed } \\
\text { meters }\end{array}$ & Age Determination \\
\hline 1 & 0 to 8.5 & Sections 1, 2, 3, 4, 5, 6: Indeterminate. \\
2 & 25.6 to 34.7 & $\begin{array}{l}\text { Sections 1, 2, 3, 4: within interval } \\
{\left[\begin{array}{l}\text { Zone P.19 } \\
\text { Zone P.18 }] ; \\
\text { Oligocene. }\end{array}\right.}\end{array}$ \\
\hline
\end{tabular}


Hole 20C (lat $28^{\circ} 31.47^{\prime} \mathrm{S}$., long $26^{\circ} 50.73^{\prime} \mathrm{W}$.) (Continued)

\begin{tabular}{|c|c|c|}
\hline Core & $\begin{array}{l}\text { Depth Below Sea Bed } \\
\text { meters }\end{array}$ & Age Determination \\
\hline 2 (Cont) & & 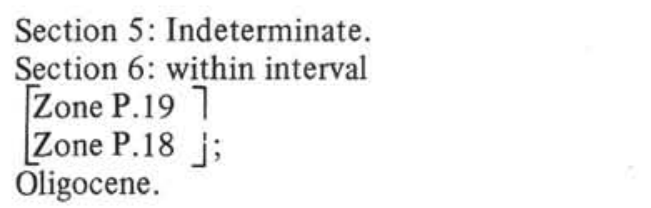 \\
\hline 3 & 36.9 to 46.0 & $\begin{array}{l}\text { Sections 1, 2, 3: Indeterminate. } \\
\text { Sections 4, 5: within interval } \\
{\left[\begin{array}{l}\text { Zone P.18 early part } \\
\text { Zone P.16 }\end{array}\right]} \\
\text { Late Eocene/basal Oligocene. } \\
\text { Section 6: Indeterminate. }\end{array}$ \\
\hline 4 & 46.9 to 56.1 & 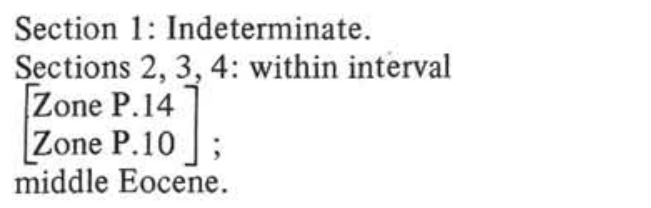 \\
\hline 5 & 57.0 to 66.1 & $\begin{array}{l}\text { Sections 1, 2, 3: within interval } \\
{\left[\begin{array}{l}\text { Zone P.11 } \\
\text { Zone P.9 }\end{array}\right]} \\
\text { lower part Middle Eocene to upper part } \\
\text { Lower Eocene. } \\
\text { Sections 4, 5: Zone P.9; } \\
\text { latest Lower Eocene. } \\
\text { Section 6: Zone P.8; } \\
\text { Lower Eocene. }\end{array}$ \\
\hline 6 & 67.1 to 72.2 & $\begin{array}{l}\text { Section 1: Zone P.8; } \\
\text { Lower Eocene. } \\
\text { Section 2: either upper Zone P.6 or lower } \\
\text { Zone P.7; } \\
\text { i.e., near Paleocene/Lower Eocene boundary. } \\
\text { Section 3: Probably Zone P.6; } \\
\text { Paleocene. } \\
\text { Section 4: Zone P.2; } \\
\text { early Paleocene but with reworked } \\
\text { Maestrichtian. } \\
\text { Section 5: } \\
\text { Late Maestrichtian. }\end{array}$ \\
\hline
\end{tabular}

Hole 21 (lat $28^{\circ} 35.0^{\prime}$ S., long $30^{\circ} 35.85^{\prime}$ W.)

\begin{tabular}{lll}
\hline Core & $\begin{array}{c}\text { Depth Below Sea Bed } \\
\text { meters }\end{array}$ & \multicolumn{1}{c}{ Age Determination } \\
\hline 1 & 20 to 29 & $\begin{array}{l}\text { Sections 1, 2, 3: within the interval } \\
\text { Zone N.20 } \\
\text { Zone N.19 }] ;\end{array}$ \\
& $\begin{array}{l}\text { Pliocene. } \\
\text { Sections 4, 5, 6: Zone N.19; } \\
\end{array}$ & early Pliocene. \\
\hline
\end{tabular}




\begin{tabular}{lll} 
Hole 21 (lat $28^{\circ} 35.0^{\prime}$ S., long $30^{\circ} 35.85^{\prime}$ W. (Continued) & \\
\hline Core & $\begin{array}{c}\text { Depth Below Sea Bed } \\
\text { meters }\end{array}$ & \multicolumn{1}{c}{ Age Determination } \\
\hline 67 to 76.0 & $\begin{array}{l}\text { Sections 1, 2: Zone P.6; } \\
\text { Late Paleocene. } \\
\text { Sections 3, 4: Zones P.6 and P.4; } \\
\text { mixed Late and Middle Paleocene mixed. } \\
\text { Sections 5, 6: Zone P.4; } \\
\text { middle Paleocene. }\end{array}$ \\
& 76 to 85.0 & $\begin{array}{l}\text { Section 1: Zone M.14; } \\
\text { Late Maestrichtian. }\end{array}$
\end{tabular}

Note: Cores 4, 5, 6, 7 and 8, show succession from Late Maestrichtian to early Campanian or ?Late Santonian.

\begin{tabular}{lcl}
\hline Hole 21A (lat $28^{\circ} 35.0^{\prime}$ S., long $\left.30^{\circ} 35.85^{\prime} \mathrm{W}.\right)$ & \\
\hline Core & $\begin{array}{c}\text { Depth Below Sea Bed } \\
\text { meters }\end{array}$ & \multicolumn{1}{c}{ Age Determination } \\
\hline 1 & 46.0 to 55.0 & $\begin{array}{l}\text { Sections 1, 2, 3: Zone P.11; } \\
\text { middle Eocene. } \\
\text { Sections 4, 5, 6: Zone P.10 or Zone P.11; } \\
\text { middle Eocene. }\end{array}$ \\
& & $\begin{array}{l}\text { Sections 1, 2, 3, 4, 5, 6: Zone P.8; } \\
\text { Lower Eocene. }\end{array}$ \\
& 61 to 70.0 & $\begin{array}{l}\text { Sections 1, 2, 3, 4, 5: Heterogenous faunas, } \\
\text { Zones P.8 to P.4 mixed; } \\
\text { (Lower Eocene/Upper Paleocene mixed). } \\
\text { Section 6: Zone P.4; } \\
\text { middle Paleocene. }\end{array}$ \\
\hline
\end{tabular}

\begin{tabular}{|c|c|c|}
\hline \multicolumn{3}{|c|}{ Hole 22 (lat $30^{\circ} 00.31^{\prime} \mathrm{S}$. , long $35^{\circ} 15.00^{\prime} \mathrm{W}$.) } \\
\hline Core & $\begin{array}{l}\text { Depth Below Sea Bed } \\
\text { meters }\end{array}$ & Age Determination \\
\hline 1 & 0 to 9.1 & $\begin{array}{l}\text { Sections 1, 2, 3, 4, 6: Zone N.22; } \\
\text { Pleistocene. }\end{array}$ \\
\hline 2 & 47 to 56.1 & $\begin{array}{l}\text { Sections 1, 2, 6: Zone N.4; } \\
\text { early Miocene. }\end{array}$ \\
\hline 3 & 104.6 to 113.8 & $\begin{array}{l}\text { Sections 1, 2, 3, 4: Zone N.3; } \\
\text { late Oligocene. } \\
\text { Sections 5, 6: Zone N.2; } \\
\text { late Oligocene. }\end{array}$ \\
\hline 4 & 133.3 to 142.4 & $\begin{array}{l}\text { Sections 1, 2: Zone N.2; } \\
\text { late Oligocene. } \\
\text { Sections 3, 4, 5, 6: Zone N.1 (=P.20); } \\
\text { late Oligocene. }\end{array}$ \\
\hline 5 & 134.2 to 242.2 & 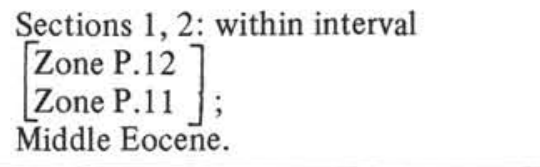 \\
\hline
\end{tabular}




\section{FAUNAS OF SAMPLES EXAMINED}

(Determinations by W.H. Blow)

\section{Hole 13}

Sample 3-13-1-1, $70 \mathrm{~cm}$ :

Rich fauna with Globorotalia $(G)$ cf. tumida, G. (G.) miocenica, G. (G.) cultrata exilis, G. (G.) cultrata menardii, G. (G.) crassula crassula, G. (G.) crassula conomiozea, G. (T.) acostaensis humerosa, G. (T.) acostaensis pseudopima, G. (T.) crassaformis crassaformis, $G$. (T.) crassaformis ronda, Sphaeroidinella dehiscens dehiscens, Pulleniatina obliquiloculata obliquiloculata, $P$. obliquiloculata $\mathrm{cf}$. praecursor, Globorotaloides hexagona hexagona, Globigerina eggeri eggeri, Globigerinoides conglobatus conglobatus, $G$. quadrilobatus (group), G. 'cf. fistulosus, G. ruber, G. elongatus. Age: Zone N.10, middle Pliocene.

Sample 3-13-1-2, 78-80 cm:

Rich fauna with Globorotalia (G.) cultrata menardii, G. (G.) cultrata exilis, G. (G.) miocenica, G. (G.) crassula conomiozea, $G$. (G.) crassula viola, $G$. (T.) acostaensis acostaensis, G. (T.) acostaensis humerosa, G. (T.) acostaensis pseudopima, $G$. (T.) ventriosa, G. (T.) crassaformis crassaformis, G. (T.) crassaformis ronda, G. (T.) obesa, Sphaeroidinella dehiscens dehiscens, Pulleniatina obliquiloculata cf. obliquiloculata, Candeina nitida nitida, Globorotaloides hexagona hexagona, Globigerina eggeri eggeri, G. decoraperta, Globigerinoides fistulosus, G. quadrilobatus (group), G. ruber, G. elongatus, Hastigerina siphonifera siphonifera and Orbulina spp.

Age: Zone N.20, middle Pliocene.

Sample 3-13-1-3, 70-72 cm:

Rich planktonic foraminiferal fauna but much affected by solution and ? bioturbation-fauna much comminuted. Fauna similar to Section 2 above.

Age: Zone N.20, middle Pliocene.

Sample 3-13-1-4, 74-76 cm:

As above.

Age: Zone N.20, middle Pliocene.

Sample 3-13-1-5, 84-86 cm:

Rich planktonic foraminiferal fauna with Globorotalia (G.) cultrata (s.l.), G. (G.) cultrata exilis, G. (G.) miocenica, $G$. (G.) crassula viola, $G$. (G.) crassula conomiozea, $G$. (T.) ventriosa, $G$. (T.) acostaensis acostaensis, G. (T.) acostaensis humerosa, G. (T.) acostaensis pseudopima, G. (T.) crassaformis crassaformis, G. (T.) crassaformis ronda, Globigerina eggeri eggeri, G. calida praecalida, G. decoraperta, G. pseudofoliata, Globigerinoides conglobatus conglobatus, G. fistulosus, G. quadrilobatus (s.l.), G. ruber, G. elongatus, Globorotaloides hexagona hexagona, Turborotalita spp., Hastigerina siphonifera siphonifera, Sphaeroidinella dehiscens dehiscens, Pulleniatina obliquiloculata cf. obliquiloculata. Age: Zone N.20, middle Pliocene.

Sample 3-13-1-6, 76-78 cm:

As above.

Age: Zone N.20, middle Pliocene.

Sample 3-13-2-1, 80-82 cm:

Fairly rich but comminuted planktonic foraminiferal fauna. Sphaeroidinella dehiscens dehiscens, Pulleniatina obliquiloculata $\mathrm{cf}$. obliquiloculata, $P$. obliquiloculata praecursor, Globorotalia (G.) cultrata exilis, $G$. (G.) cultrata menardii, G. (G.) cf. multicamerata, Globigerinoides conglobatus conglobatus, $G$. ruber, Globigerina calida praecalida, G. pseudofoliata, $G$. decoraperta, Globorotalia (T.) acostaensis acostaensis, G. (T.) acostaensis humerosa, G. (T.) acostaensis cf. pseudopima, Globigerina eggeri eggeri, etc.

Age: Zone N.20, middle Pliocene.

Sample 3-13-2-2, 80-82 cm:

Fauna fragmentary only, no recognizable planktonic foraminiferal species present.

Age: None.

Sample 3-13-2-3, 75-77 cm:

Rich, mainly fragmentary fauna with Globorotalia $(G$. tumida tumida, G. (G.) cultrata (s.l.), G. (G.) miocenica, Sphaeroidinella dehiscens dehiscens, Sphaeroidinellopsis subdehiscens paenedehiscens, $S$. cf, seminulina (s.l.). Age: ?Zone N.19.

Sample 3-13-2-4, 73-75 cm:

No planktonic foraminiferal fauna.

Age: None.

Sample 3-13-2-5, 73-75 cm:

No planktonic foraminiferal fauna; micronodules of manganese.

Age: None.

Sample 3-13-2-6, 78-80 cm:

No planktonic foraminiferal fauna; micronodules of manganese and fish teeth.

Age: None.

Sample 3-13-3-1, 99-101 cm:

Rich, radiolarian fauna; no planktonic foraminifera. Age: No planktonic foraminiferal determination.

Sample 3-13-3-3, 75-77 cm:

As above.

Sample 3-13-3-6, 72-74 cm:

As above. 


\section{Hole 13A}

Sample 3-13A-1-1, 119-121 cm:

Fairly rich radiolarian fauna; no planktonic foraminiferal species present.

Age: No planktonic foraminiferal determination.

Sample 3-13A-2-1, 128-130 cm:

No planktonic foraminiferal fauna, nearly barren. Age: None.

Sample 3-13A-3-1, 105-107 cm:

Virtually barren.

Age: None.

Sample 3-13A-3-2, 76-78 cm:

Echinoid fragments only.

Age: None.

Sample 3-13A-3-3, 75-77 cm:

Virtually barren.

Age: None.

Sample 3-13A-3-4, 80-82 cm:

Very poor benthonic foraminiferal fauna with a specimen of Globorotalites sp.

Age: Upper Cretaceous.

Sample 3-13A-4-1, 78-82 cm:

Inoceramus.

\section{Hole 14}

Sample 3-14-1A-1, 75-77 cm:

Globorotalia (Turborotalia) sp, Globorotalia (T.) cf. siakensis, Globigerinita stainforthi stainforthi, Globigerinita dissimilis dissimilis, G. unicava unicava, G. incrusta, Globigerinoides ef. subquadratus, G. quadrilobatus immaturus, G. quadrilobatus trilobus, Globigerina woodi (s.l.), Globoquadrina dehiscens cf. advena. Age: Probably Zone N.6 (basal part?), Aquitanian, early Miocene.

Sample 3-14-1A-2, 75-77 cm:

As above.

Age: Probably Zone N.6 (basal part?), Aquitanian, early Miocene.

Sample 3-14-1A-3, 75-77 cm:

Rather poor, mainly fragmentary planktonic foraminiferal fauna with Globigerinita dissimilis dissimilis, G. dissimilis ciperoensis, G. incrusta, Globigerinoides quadrilobatus immaturus, G. quadrilobatus trilobus.

Age: Zone N.5 or Zone N.6, early Miocene, Aquitanian.

Sample 3-14-1A-4, 75-77 cm:

Fragmentary fauna, no specifically determinable specimens.

Age: None.
Sample 3-14-1A-5, 75-77 cm:

Fairly rich planktonic foraminiferal fauna. Globorotalia (T.) cf. siakensis, G. (T.) clemenciae, Globigerinita dissimilis dissimilis, G. dissimilis ciperoensis, G. unicava unicava, G. incrusta, Globigerina juvenilis, G. praebulloides praebulloides, Globigerinoides quadrilobatus immaturus, G. cf. subquadratus, Globoquadrina dehiscens dehiscens.

Age: Zone N.5 (?upper part), early Miocene, Aquitanian.

Sample 3-14-1A-6, 74-75 cm:

Poor, mainly fragmentary fauna with Globigerinoides quadrilobatus immaturus, Globigerinita dissimilis ciperoensis, G. incrusta, Globigerina juvenilis.

Age: Zone N.5, early Miocene, Aquitanian.

Sample 3-14-2-1, 74-76 cm:

Fairly rich planktonic foraminiferal fauna with Globorotalia (T.) kugleri, G. (T.) pseudokugleri, G. (T.) siakensis, Globigerinoides quadrilobatus immaturus, $G$. quadrilobatus primordius, Globigerinita dissimilis ciperoensis, $G$. dissimilis dissimilis, G. unicava unicava, $G$. stainforthi praestainforthi, $G$. incrusta, Globigerina praebulloides occlusa, G. juvenilis, Globoquadrina dehiscens praedehiscens.

Age: Zone N.4, Aquitanian, early Miocene.

Sample 3-14-2-2, 75-77 cm:

Rich planktonic foraminiferal fauna with Globorotalia (T.) kugleri, G. (T.) pseudokugleri, G. (T.) siakensis, Globoquadrina altispira globularis, G. dehiscens praedehiscens, Globigerinoides quadrilobatus immaturus, $G$. quadrulobatus primordius, Globigerina praebulloides occlusa, G. juvenilis, Globigerinita stainforthi praestainforthi, G. incrusta, G. dissimilis ciperoensis, G. dissimilis dissimilis, $G$. unicava unicava.

Ảge: Zone N.4, Aquitanian, early Miocene.

Sample 3-14-2-3, 75-77 cm:

As above.

Sample 3-14-2-4, 75-77 cm:

Rich planktonic foraminiferal fauna with Globigerinoides quadrilobatus primordius (rare), Globigerina praebulloides occlusa, $G$. woodi woodi, $G$. venezuelana, G. juvenilis, Globigerinita incrusta, G. dissimilis dissimilis, G. dissimilis ciperoensis, G. unicava unicava, Globoquadrina dehiscens praedehiscens, G. altispira globularis, Globorotalia (T.) kugleri, G. (T.) pseudokugleri, $G$. (T.) cf. mendacis, $G$. (T.) cf. siakensis.

Age: Zone N.4.

Sample 3-14-2-5, $75-77 \mathrm{~cm}$ :

As above. 
Sample 3-14-3-1, $73-75 \mathrm{~cm}$ :

Fairly rich planktonic foraminiferal fauna with Globigerina praebulloides occlusa, $G$. venezuelana, $G$. angustiumbilicata, Globorotalia (T.) cf. mendacis, G. (T.) gemma, G. (T.) clemenciae, G. (T.) siakensis, $G$. (T.) clemenciae, Globigerinita dissimilis dissimilis, $G$. dissimilis ciperoensis, G. unicava unicava, Globoquadrina dehiscens praedehiscens.

Age: Probably Zone N.3 (uppermost part), Oligocene.

Sample 3-14-3-2, $73-75 \mathrm{~cm}$ :

As above.

Sample 3-14-3-3, $73-75 \mathrm{~cm}$ :

Rich planktonic foraminiferal fauna with Globigerina praebulloides occlusa, G. angulisuturalis (rare), G. ouachitaensis cf. ciperoensis (rare), G. juvenilis, Globorotalia (T.) siakensis, G. (T.) cf. siakensis (of Blow, 1969), G. (T.) clemenciae, G. (T.) gemma, G. (T.) permicra, Globoquadrina altispira globularis, $G$. cf. baroemeonensis, $G$. dehiscens praedehiscens, Globigerina venezuelana, $G$. cf. tripartita, Globigerinita dissimilis (s.l.), G. unicava unicava, Globorotaloides cf. suteri. Age: Zone N.3, Oligocene.

Sample 3-14-3-4, $72-74 \mathrm{~cm}$ :

As above.

Sample 3-14-3-5, $72-74 \mathrm{~cm}$ :

As above.

Sample 3-14-3-6, 75-77 cm:

Rich planktonic foraminiferal fauna with taxa as listed for Sample 3-14-3-3, $73-75 \mathrm{~cm}$ plus specimens referable to Globigerina sellii.

Age: Zone N.3, Oligocene.

Sample 3-14-4-1, 29-31 cm:

Globigerinita unicava unicava, G. dissimilis dissimilis, G. pera, Globorotaloides suteri, Globorotalia (T.) cf. siakensis (of Blow, 1969), G. (T.) opima nana, G. (T.) opima cf. opima, G. (T.) clemenciae, G. (T.) gemma, $G$. (T.) permicra, Globigerina prasaepis, G. tripartita, $G$. galavisi, specimens ex interc G. galavisi-Globoquadrina altispira globularis, Globigerina sellii, Globigerina cf. winkleri.

Age: Zone N.2, Oligocene.

Sample 3-14-4-2, 103-105 cm:

As above.

Sample 3-14-4-3, $75-77 \mathrm{~cm}$ :

Rich planktonic foraminiferal fauna with Globorotalia (T.) opima opima, G. (T.) opima nana, G. (T.) cf. siakensis (of Blow, 1969), G. (T.) gemma, G. (T.) permicra, G. (T.) clemenciae, Globigerina prasaepis, G. cf. tripartita, G. sellii, G. galavisi, Globorotaloides suteri, Age: Zone N.2, Oligocene.
Sample 3-14-5-1, 73-75 cm:

Fairly rich foraminiferal fauna with Globigerina galavisi, G. tripartita, G. sellii, G. ampliapertura, G. prasaepis, Globorotalia (T.) opima nana, G. (T.) permicra, G. (T.) gemma, G. (T.) cf. siakensis (of Blow, 1969), Globorotaloides suteri, Globigerinita unicava unicava, G. dissimilis dissimilis.

Age: Zone N.1 (=P.20), Oligocene.

Sample 3-14-5-2, $72-74 \mathrm{~cm}$ :

As above.

Age: Zone N.1 (=P.20), Oligocene.

Sample 3-14-5-3, 75-77 cm:

As above.

Age: Zone N.1 (=P.20), Oligocene.

Sample 3-14-5-4, 73-75 cm:

Globorotalia (Turborotalia) cf. increbescens, G. (T.) increbescens, G. (T.) cf. siakensis (of Blow, 1969), Globigerina prasaepis, G. galavisi, G. cf. sellii, G. tripartita, G. ampliapertura, Globigerinita dissimilis dissimilis, $G$. unicava unicava, Globorotaloides suteri.

Age: Zone N.1 (=P.20), Oligocene.

Sample 3-14-5-5, 76-78 cm:

Globorotalia (T.) cf. increbescens, G. (T.) gemma, G. (T.) permicra, Globigerina ampliapertura (v. rare), G. anguliofficinalis (v. rare), G. ouachitaensis cf. ciperoensis, G. cf. tripartita, G. prasaepis, G. galavisi, Globigerinita unicava unicava, G. unicava primitiva, G. dissimilis dissimilis, Globorotaloides suteri.

Age: Zone N.1 (=P.20), Oligocene.

Sample 3-14-5-6, $72-74 \mathrm{~cm}$ :

As above.

Age: Zone N.1 (=P.20), Oligocene.

Sample 3-14-6-1, 85-87 cm:

Globigerina angiporoides, Globigerina ampliapertura, Globigerina galavisi, G. tripartita, G. cf. sellii, G. prasaepis, Globorotalia (T.) cf. increbescens. G. (T.) increbescens, G. (T.) opima cf. opima, G. (T.) opima nana, Globorotalia (T.) gemma, G. (T.) permicra, Globigerinita dissimilis dissimilis, G. unicava cf. unicava, G. unicava primitiva, G. pera, G. martini scandretti.

Age: Within interval of $\left[\begin{array}{l}\text { Zone N.1 (=P.20) early part } \\ \text { Zone P.19, later part }\end{array}\right]$, Oligocene (see Note 2 below).

Sample 3-14-6-2, $77-79 \mathrm{~cm}$ :

Globigerina prasaepis, Globigerina ampliapertura, G. angiporoides, G. galavisi, G. tripartita, G. cf. sellii, Globorotalia (T.) increbescens, G. (T.) cf. increbescens, G. (T.) opima nana, G. (T.) opima cf. opima, G. (T.) 
gemma, G. (T.) permicra, Globigerinita pera, Globigerinita dissimilis dissimilis, Globigerinita unicava cf. unicava, G. unicava primitiva, Globigerina senilis, Globigerina cf. anguliofficinalis, $G$. officinalis, $G$. angustiumbilicata, Globigerinita martini scandretti, Globorotaloides suteria.

Age: Within interval of $\left[\begin{array}{l}\text { Zone N.1 (=P.20) early part } \\ \text { Zone P.19, later part }\end{array}\right]$, Oligocene (See Note 2 below).

Sample 3-14-6-3, 77-79 cm:

As above but also with specimens referable to Globigerina tapuriensis.

Age: Within the interval $\left[\begin{array}{l}\text { Zone N.1 (=P.20) early part } \\ \text { Zone P.19, later part }\end{array}\right]$, Oligocene (see Note 2, below).

Sample 3-14-6-4, 84-86 cm:

As for Sample 3-14-6-3, 77-79 cm:

Age: Within the interval $\left[\begin{array}{l}\text { Zone N.1 (=P.20) early part } \\ \text { Zone P.19, later part }\end{array}\right]$, Oligocene (see Note 2 below).

Sample 3-14-6-5, 77-79 cm:

As for Sample 3-14-6-3, 77-79 cm but with a specimen referable to Globigerina gortanii gortanii.

Age: Within the interval $\left[\begin{array}{l}\text { Zone N.1 (=P.20), early part } \\ \text { Zone P.19. later part }\end{array}\right]$, Oligocene (see Note 2 below).

Sample 3-14-6-6, 77-79 cm:

As for Sample 3-14-6-3, 77-79 cm.

Age: Within the interval $\left[\begin{array}{l}\text { Zone N.1 (=P.20), early part } \\ \text { Zone P.19, later part }\end{array}\right]$, Oligocene (see Note 2 below).

Sample 3-14-7-1, 67-78 cm:

Globigerina cf. sellii, $G$. sellii ex interc $G$. tapuriensis, G. tapuriensis, G. pseudoampliapertura, G. prasaepis, $G$. cf. pseudovenezuelana, $G$. angiporoides, $G$. galavisi, G. ampliapertura, $G$. senilis, Globigerinita dissimilis dissimilis, G. pera, G. unicava primitiva, Globorotalia (T.) gemma, G. (T.) permicra, Globorotaloides suteri.

Age: Zone P.19, probably early part, Oligocene (see

Note 3 below).

Sample 3-14-7-2, 67-78 cm:

Globigerina tapuriensis, G. pseudoampliapertura, G. prasaepis, G. pseudovenezuelana, G. tripartita, G. angiporoides, G. galavisi, G. gortanii gortanii, G. senilis, Globigerinita unicava primitiva, Globorotaloides suteri, Globorotalia (T.) permicra, G. (T.) gemma, G. (T.) increbescens.

Age: Zone P.19, probably early part, Oligocene (see Note 3 below).

Sample 3-14-7-3, 76-78 cm:

As above.
Sample 3-14-7-4, 76-78 cm:

Globigerina tapuriensis, $G$. cf. sellii, G. prasaepis, $G$. cf. pseudovenezuelana, G. angiporoides, G. pseudoampliapertura, $G$. praebulloides leriyi, $G$. officinalis, $G$. cf. anguliofficinalis, Globorotalia (T.) cf. increbescens, $G$. (T.) opima nana, G. (T.) permicra, G. (T.) gemma, Globorotaloides suteri, Globigerinita martini martini, G. martini scandretti, G. dissimilis dissimilis, G. unicava primitiva, G. pera, Pseudohastigerina barbadoensis, Cassigerinella eocaenica.

Age: Zone P.19, early part, Oligocene (see Note 4 below).

Sample 3-14-7-5, 72-74 cm:

Globigerina tapuriensis, G. prasaepis, G. cf. pseudovenezuelana, $G$. galavisi, G. tripartita, G. angiporoides, Cassigerinella eocaenica, $C$. chipolensis, Pseudohastigerina barbadoensis, Globorotalia (T.) gemma, G. (T.) permicra, G. (T.) cf. increbescens, $G$. (T.) opima nana, Globigerinita martini (s.l.), Globigerinita pera, G. unicava primitiva.

Age: Probably Zone P.18 (Oligocene) (see Note 4 below).

Sample 3-14-7-6, 72-74 cm:

Globigerina ampliapertura, G. angiporoides, G. prasaepis, $G$. pseudovenezuelana, G. galavisi, G. pseudoampliapertura, G. cf. tripartita, G. tapuriensis, G. gortanii gortanii, Cassigerinella chipolensis, C. cf. eocaenica, Globigerinita martini martini, G. martini scandretti, $G$. pera, G. dissimilis dissimilis, G. unicava primitiva, Globorotaloides suteri, Globorotalia (T.) cf. increbescens, G. (T.) increbescens, G. (T.) opima nana, G. (T.) permicra, G. (T.) gemma, Pseudohastigerina barbadoensis.

Age: Zone P.18, Oligocene.

Sample 3-14-8-1, 80-82 cm:

Fauna mainly comminuted Globigerina anguliofficinalis, G. gortanii gortanii, G. angiporoides, G. prasaepis, Pseudohastigerina barbadoensis, Globorotalia (T.) increbescens, G. (T.) gemma, G. (T.) permicra.

Age: Probably Zone P.18, Oligocene.

Sample 3-14-8-2, $72-74 \mathrm{~cm}$ :

Globigerina anguliofficinalis, $G$, angiporoides, $G$. galavisi, G. prasaepis, G. tripartita, G. tapuriensis, Globigerinita unicava primitiva, G. pera, G. martini scandretti, G. martini martini, Pseudohastigerina barbadoensis, Globorotalia (T.) permica, G. (T.) gemma.

Age: Zone P.18, Oligocene.

Sample 3-14-8-3, 80-82 cm:

As above.

Sample 3-14-8-5, 48-50 cm:

As above. 
Sample 3-14-8-6, 148-150 cm:

Globigerina prasaepis, G. pseudovenezuelana, G. ampliapertura, G. pseudoampliapertura, G. tripartita, G. galavisi, G. cf. winkleri, G. gortanii gortanii, G. angiporoides, Globigerinita dissimilis dissimilis, G. martini martini, G. martini scandretti, G. pera, G. unicava primitiva, Globorotalia (T.) gemma, G. (T.) permicra, Pseudohastigerina barbadoensis, Globorotalia (T.) opima nana, $G$. (T.) increbescens.

Age: Early Zone P.18 (earliest Oligocene).

Sample 3-14-9-2, $72-74 \mathrm{~cm}$ :

Globigerina ampliapertura, G. pseudoampliapertura, $G$. tapuriensis, G. galavisi, G. tripartita, G. prasaepis, G. pseudovenezuelana, G. gortanii gortanii, G. angiporoides, Globigerinita pera, G. dissimilis dissimilis, G. martini martini, G. martini scandretti, Globorotalia (T.) cf. increbescens, $G$. (T.) increbescens, $G$. (T.) opima nana, $G$. (T.) permicra, G. (T.) gemma, Pseudohastigerina barbadoensis.

Age: Probably early Zone P.18, earliest Oligocene.

Sample 3-14-9-3, 148-150 cm:

Poor planktonic foraminiferal fauna with Globigerina pseudovenezuelana, Globigerinita pera, G. dissimilis dissimilis, G. unicava primitiva, G. martini martini, $G$. martini scandretti, Pseudohastigerina barbadoensis.

Age: Early Zone P.18 or latest Zone P.17 (earliest Oligocene or latest Eocene).

Sample 3-14-9-4, 73-75 cm:

As above.

Sample 3-14-9-5, 75-77 cm:

Globigerina ampliapertura, G. pseudovenezuelana, $G$. pseudoampliapertura, $G$. cf. prasaepis, G. angiporoides, G. tripartita, G. galavisi, Globorotalia (T.) permicra, G. (T.) gemma, G. (T.) increbescens, Globigerinita martini martini, G. martini scandretti, G. pera, G. unicava primitiva, Pseudohastigerina barbadoensis.

Age: Probably Zone P.17, latest Eocene (see Note 5 below).

Sample 3-14-9-6, 86-88 cm:

Fairly rich planktonic foraminiferal fauna with Globigerina ampliapertura, G. pseudoampliapertura, G. galavisi, G. angiporoides, G. tripartita, Globorotalia $\mathrm{cf}$. increbescens, G. (T.) increbescens, G. (T.) permicra, G. (T.) gemma, Globigerinita martini martini, G. martini scandretti, G. pera, G. unicava primitiva, Pseudohastigerina micra, $P$. barbadoensis.

Age: Zone P.17, latest Eocene (see Note 5 below).

Notes concerning the succession recognized at Hole 14:

1. Globigerina angulisuturalis is virtually absent in the interval ascribed to Zone N.2 (=P.21) and Zone N.3 (=P.22).
2. Neither Cassigerinella chipolensis nor C. eocaenica occurs in any significant numbers throughout the succession penetrated. Furthermore, it would appear that Pseudohastigerina is likewise excluded by facies for the latest part of its range. The Zone N.1 (=P.20)/Zone P.19 boundary can be (and has been) fixed on the extinction horizons of Globigerinita martini (s.l.) and Globigerina angiporoides. The former tax on has been recorded elsewhere from the lowest part of Zone N.1 (=P.20) but, previously, $G$. angiporoides has not been seen above the interval of Zone P.19. It would now appear that both these taxa become extinct not far from the Zone N.1 (=P.20)/Zone P.19 boundary, but probably just subsequent to the boundary. Hence Core 6 is considered to be within the interval of

$$
\left[\begin{array}{l}
\text { Zone N.1 (=P.20) early part } \\
\text { Zone N.19 latest part }
\end{array}\right] \text {. }
$$

3. The definite assignment of Zone P.19 can be made on the presence of Globigerina tapuriensis notwithstanding the absence of Pseudohastigerina barbadoensis.

4. Pseudohastigerina barbadoensis occurs rarely in Core 7, Section 4, with Cassigerinella eocaenica and this indicates the proximity of the Zone P.19/ Zone P.18 boundary.

5. Only the very youngest part of the Eocene is represented in Core 9, Sections 5 and 6.

\section{Hole 15}

Sample 3-15-1-1, 77-79 cm:

Rich planktonic foraminiferal fauna with Globorotalia (G.) cultrata menardii, $G$. (G.) tumida tumida, G. (G.) truncatulinoides truncatulinoides, $G$. (G.) truncatulinoides pachytheca, G. (G.) crassula (s.l.), Globorotalia (Turborotalia) inflata, G. (T.) crassaformis crassaformis, $G$. (T.) crassaformis ronda, Globigerina oscitans, $G$. eggeri eggeri, Globigerinoides conglobatus conglobatus, G. quadrilobatus (s.l.), Pulleniatina obliquiloculata obliquiloculata, Sphaeroidinella dehiscens dehiscens, Turborotalita spp.

Age: Zone N.22, Pleistocene.

Sample 3-15-1-2, 71-73 cm:

As above.

Sample 3-15-1-3, 80-82 cm:

As above.

Sample 3-15-1-4, 80-82 cm:

As above.

Sample 3-15-1-5, $72-74 \mathrm{~cm}$ :

As above. 
Sample 3-15-1-6, 76-78 cm:

As above.

Sample 3-15-2-1, 72-74 cm:

Rich planktonic foraminiferal fauna with Globorotalia (G.) cultrata menardii, G. (G.) cultrata exilis, $G$. (G.) crassula crassula, G. (G.) crassula viola, $G$. (T.) crassaformis crassaformis, $G$. (T.) crassaformis ronda, $G$. (T.) inflata, G. (T.) puncticulata, G. (G.) truncatulinoides truncatulinoides (very rare and phylogenetically primitive), G. (T.) cf. tosaensis (s.l.), Globigerinoides elongatus, G. conglobatus conglobatus, G. ruber, G. quadrilobatus (s.l.), Sphaeroidinella dehiscens dehiscens, Candeina nitida nitida, Orbulina spp.

Age: Basal Zone N.22, earliest Pleistocene.

Sample 3-15-2-2, $72-74 \mathrm{~cm}$ :

As above but no specimens referable to $G$. (G.) truncatulinoides (s.l.) or to G. (T.) tosaensis (s.l.).

Age: ?Zone N.21, latest Pliocene.

Sample 3-15-2-3, 76-78 cm:

Rich planktonic foraminiferal fauna with Globorotalia (G.) cultrata menardii, G. (G.) cultrata exilis, G. (G.) crassula crassula, G. (G.) crassula viola, $G$. (T.) crassaformis crassaformis, $G$. (T.) crassaformis ronda, $G$. (T.) ventriosa, G. (T.) puncticulata, G. (T.) inflata, Globigerinoides conglobatus conglobatus, G. elongatus, G. ruber, Globigerina eggeri eggeri, G. bulloides bulloides, G. bulloides cf. apertura, Hastigerina siphonifera siphonifera, Sphaeroidinella dehiscens dehiscens, Candeina nitida nitida, Orbulina spp.

Age: ?Zone N.21, late Pliocene.

Sample 3-15-2-4, $72-74 \mathrm{~cm}$ :

Same as above with Globigerina falconensis.

Age: ?Zone N.21, late Pliocene.

Sample 3-15-2-5, $72-74 \mathrm{~cm}$ :

Rich planktonic foraminiferal fauna with Globorotalia (G.) crassula crassula, G. (G.) crassula conomiozea, Globorotalia (T.) crassaformis crassaformis, G. (T.) crassaformis ronda, G. (T.) inflata, G. (T.) acostaensis acostaensis, G. (T.) trigonula, G. (T.) obesa, G. (T.) cf. ventriosa, Globigerinoides conglobatus conglobatus, $G$. elongatus, G. quadrilobatus (s.l.) (group), Globigerina decoraperta, G. falconensis, G. parabulloides, G. quadrilatera, Hastigerina siphonifera siphonifera, Sphaeroidinella dehiscens dehiscens, Turborotalita spp.

Age: Within $\left[\begin{array}{l}\text { Zone N.21 } \\ \text { Zone N.20 }\end{array}\right]$ interval, middle to late Pliocene.

Sample 3-15-2-6, 77-79 cm:

As above.

Sample 3-15-3-1, 76-78 cm:

Rich planktonic foraminiferal fauna with Globorotalia
(G.) cultrata exilis, G. (G.) margaritae, G. (G.) miozea cibaoensis, G. (G.) crassula crassula, G. (G.) crassula conomiozea, $G$. (T.) crassaformis crassaformis, $G$. (T.) crassaformis ronda, $G$. (T.) scitula scitula, $G$. (T.) acostaensis acostaensis, G. (T.) cf. subscitula, Globigerina falconensis, G. decoraperta, G. quadrilatera, G. parabulloides, Globigerinoides conglobatus conglobatus, G. elongatus, $G$. cf. ruber, Globoquadrina altispira altispira, G. altispira cf. globosa, G. dehiscens dehiscens, Sphaeroidinellopsis seminulina (s.l.), G. subdehiscens paenedehiscens, Sphaeroidinella dehiscens dehiscens forma immatura, Candeina nitida nitida.

Age: Zone N.19, early part, Early Pliocene (see Note below).

Sample 3-15-3-2, 76-78 cm:

As above.

Sample 3-15-3-3, $72-74 \mathrm{~cm}$ :

As above.

Sample 3-15-3-4, $77-79 \mathrm{~cm}$ :

As above.

Sample 3-15-3-5, 77-79 cm:

Rich planktonic foraminiferal fauna with Globorotalia (G.) cultrata exilis, G. (G.) margaritae, G. (G.) miozea cibaoensis, G. (T.) crassaformis crassaformis, G. (T.) crassaformis ronda, $G$. (T.) acostaensis acostaensis, $G$. (T.) cf. subscitula, G. (T.) scitula scitula, G. (G.) crassula crassula, G. (G.) crassula conomiozea, Globoquadrina altispira altispira, $G$. dehiscens dehiscens, Sphaeroidinellopsis subdehiscens paenedehiscens, $S$. seminulina seminulina, Candeina nitida nitida, Hasterigerina pelagica, $H$. siphonifera siphonifera, Globigerina decoraperta, $G$. parabulloides, G. falconensis, Globigerinoides conglobatus conglobatus, G. elongatus, G. bollii.

Age: Zone N.18, upper part.

Sample 3-15-3-6, 76-78 cm:

As above.

Sample 3-15-4-1, $72-74 \mathrm{~cm}$ :

Fairly rich planktonic foraminiferal fauna but one which is not well diversified with $G$. (T.) subscitula, Globorotalia (G.) miozea cf. cibaoensis, G. (G.) crassula crassula, $G$. (G.) crassula conomiozea, G. (T.) crassaformis crassaformis, $G$. (T.) crassaformis ronda, Globoquadrina altispira altispira, G. cf. dehiscens dehiscens, Sphaeroidinellopsis subdehiscens (s.l.), Globigerina decoraperta, Globigerinoides elongatus, G. quadrilobatus (group), $G$. bollii, Orbulina spp., $G$. nepenthes.

Age: ?Zone N.18.

Sample 3-15-4-2, 72-74 cm:

Rich planktonic foraminiferal fauna with Globorotalia nepenthes, G. decoraperta, G. falconensis, G. parabulloides, G. quadrilatera, Globorotalia (G.) miozea cibaoensis, $G$. (G.) crassula crassula, G. (T.) acostaensis 
humerosa, G. (T.) acostaensis acostaensis, Globigerinoides conglobatus conglobatus, G. elongatus, G. quadrilobatus (group), G. bolli, Orbulina spp., Sphaeroidinellopsis subdehiscens (s.l.), Globoquadrina altispira altispira.

Age: Zone N.18.

Sample 3-15-4-3, 76-78 cm:

As above.

Sample 3-15-4-4, 78-80 cm:

As above.

Sample 3-15-4-5, 76-78 cm:

As above.

Sample 3-15-4-6, 77-79 cm:

As above.

Sample 3-15-5-1, 77-79 cm:

Rich planktonic foraminiferal fauna much restricted as to species diversification, Globigerina nepenthes (abundant), Globigerina falconensis (rare), Globorotalia (G.) miozea (s.l.), Globoquadrina altispira altispira (rare), Globigerinoides bollii, Orbulina spp., Sphaeroidinellopsis subdehiscens subdehiscens, Globorotaliodes hexagona variabilis (rare), Globigerinita n.sp. (cf. parvula).

Age: Probably Zone N.17, Late Miocene.

Sample 3-15-5-2, 76-78 cm:

As above, but with $G$. (T.) crassaformis ronda.

Age: Probably Zone N.17, Late Miocene.

Sample 3-15-5-3, 76-78 cm:

As above.

Age: Probably Zone N.17.

Sample 3-15-5-4, 76-78 cm:

As above plus Globigerinoides ruber.

Age: Probably Zone N.17.

Sample 3-15-5-5, $72-74 \mathrm{~cm}$ :

As above.

Age: Probably Zone N.17.

Sample 3-15-5-6, 77-79 cm:

Rich planktonic foraminiferal fauna with a biofacies change to that seen above and with slightly more species diversification. Globigerina nepenthes, G. praebulloides occlusa, G. falconensis, Globigerinoides conglobatus cf. conglobatus, G. ruber, G. quadrilobatus (group), Globorotalia (G.) cultrata menardii (rare), G. (G.) miozea cf. cibaoensis, Sphaeroidinellopsis subdehiscens subdehiscens, $S$. seminulina seminulina.

Age: Probably Zone N.17.

Sample 3-15-6-1, 70-72 cm:

Poor fragmentary planktonic foraminiferal fauna with
Globigerina nepenthes, Globigerinoides bollii, Sphaeroidinellopsis subdehiscens subdehiscens.

Age: Within interval $\left[\begin{array}{l}\text { Zone N.17 } \\ \text { Zone N.14 }\end{array}\right]$, Miocene.

Sample 3-15-6-2, 77-79 cm:

As above.

Sample 3-15-6-3, 71-76 cm:

As above.

Sample 3-15-6-4, 72-74 cm:

As above, but with benthonic foraminifera and micronodules of manganese.

Sample 3-15-6-5, 76-78 cm:

Fairly rich, altered and partially fragmentary planktonic foraminiferal fauna. Globigerina nepenthes, Sphaeroidinellopsis subdehiscens subdehiscens, S. seminulina seminulina, Globorotalia (G.) miozea miozea, Globoquadrina dehiscens advena, Globigerinoides bollii, G. quadrilobatus immaturus, Globorotalia (T.) cf. mayeri. Age: Probably Zone N.15, Middle Miocene.

Sample 3-15-6-6, 77-79 cm:

Rather poor planktonic foraminiferal dwarfed fauna: Globoquadrina dehiscens dehiscens, $G$. dehiscens advena, Sphaeroidinellopsis subdehiscens subdehiscens, Globigerina cf. nepenthes (small), G. juvenilis, Globigerinita incrusta, G. parvula, Globigerinoides bollii, $G$. (T.) continuosa.

Age: Probably Zone N.15, Middle Miocene.

Sample 3-15-7-1, 76-78 cm:

Rich planktonic foraminiferal fauna with Globigerina nepenthes (v. rare), G. falconensis, G. juvenilis, Globigerinoides parkerae, G. bollii, G. quadrilobatus (group), Globoquadrina dehiscens advena, ,G. dehiscens dehiscens, Globigerinita incrusta, G. ambitacrena, G. parvula, Sphaeroidinellopsis subdehiscens subdehiscens, $S$. seminulina seminulina, Globorotalia $(G$.) miozea miozea, G. (T.) continuosa.

Age: Probably Zone N.15, Middle Miocene.

Sample 3-15-7-2, 76-78 cm:

As above.

Sample 3-15-7-3, 72-74 cm:

As above.

Sample 3-15-7-4, 77-79 cm:

As above.

Sample 3-15-7-5, 72-74 cm:

As above. 
Sample 3-15-7-6, 76-78 cm:

As above but with $G$. (G.) cultrata cf. cultrata and Globigerina parabulloides.

Age: Probably Zone N.15, middle Miocene.

Sample 3-15-8-1, 148-150 cm:

Rich planktonic foraminiferal fauna with Globorotalia (T.) siakensis, Globigerinita dissimilis dissimilis, G. dissimilis ciperoensis, G. unicava unicava, Globigerinoides subquadratus, G. quadrilobatus trilobus, G. quadrilobatus immaturus, Globigerinita incrusta, Globoquadrina dehiscens dehiscens.

Age: Zone N.6 lower part or ?Zone N.5, upper part Early Miocene (see Note below).

Sample 3-15-8-2, $72-74 \mathrm{~cm}$ :

Very rich planktonic foraminiferal fauna with Globigerinita dissimilis dissimilis, $G$. dissimilis ciperoensis, $G$. unicava unicava, G. stainforthi stainforthi, G. incrusta, Globigerinoides quadrilobatus altiaperturus, G. quadrilobatus cf. quadrilobatus, G. quadrilobatus trilobus, G. quadrilobatus immaturus, G. subquadratus, Globigerina woodi woodi, G. woodi connecta, G. praebulloides occlusa, G. juvenilis, Globorotalia (T.) siakensis, $G$. (T.) obesa, Globoquadrina dehiscens dehiscens, $G$. dehiscens $\mathrm{cf}$. praedehiscens.

Age: Zone N.6, lower part of ?Zone N.5, upper part Early Miocene (see Note below).

Sample 3-15-8-3, $77-79 \mathrm{~cm}$ :

As above.

Age: As above (see Note below).

Sample 3-15-8-4, $77-79 \mathrm{~cm}$ :

As above.

Age: As above (see Note below).

Sample 3-15-8-5, 77-79 cm:

As above.

Age: As above (see Note below).

Sample 3-15-8-6, 77-79 cm:

As above.

Age: As above (see Note below).

Sample 3-15-9-1, 77-79 cm:

Rich planktonic foraminiferal fauna with Globorotalia (G.) miozea miozea (phylogenetically primitive), $G$. (T.) scitula praescitula, $G$. (T.) scitula cf. gigantea, $G$. (T.) siakensis, Globorotaloides cf. hexagona variabilis, Globigerinoides subquadratus, G. quadrilobatus trilobus, G. quadrilobatus immaturus, Globigerina venezuelana, $G$. falconensis, $G$. praebulloides occlusa, $G$. woodi woodi, Globoquadrina dehiscens dehiscens, G. dehiscens advena, Globigerinita incrusta, Globoquadrina altispira globosa, G. altispira cf. altispira, Sphaeroldinellopsis seminulina (primitive).

Age: Zone N.7, Early Miocene. (see Note below).
Sample 3-15-9-2, 76-78 cm:

As above.

Age: As above (see Note below).

Sample 3-15-9-3, 76-78 cm:

As above.

Age: As above (see Note below).

Sample 3-15-9-4, 76-78 cm:

Rich planktonic foraminiferal fauna with Globigerinita dissimilis dissimilis (v. rare), G. unicava unicava (v. rare), Globorotalia (T.) scitula praescitula, G. (T.) scitula cf. gigantea, G. (G.) cf. miozea miozea, G. (T.) cf. birnageae, $G$. (T.) siakensis, Globigerinoides quadrilobatus trilobus, G. quadrilobatus immaturus, G. subquadratus, Globigerinita incrusta, Globigerina cf. falconensis, G. praebulloides (s.l.), G. juvenilis, G. woodi woodi, Globoquadrina altispira cf. altispira, G. dehiscens dehiscens, G. dehiscens advena, Globorotaloides cf. hexagona variabilis.

Age: Latest Zone N.6, Early Miocene. (see Note below).

Sample 3-15-9-5, $77-79 \mathrm{~cm}$ :

Fairly rich planktonic foraminiferal fauna with Globigerinita dissimilis dissimilis, $G$. dissimilis ciperoensis, $G$. unicava unicava, $G$. incrusta, Globoquadrina dehiscens dehiscens, $G$. dehiscens cf. advena, G. altispira cf. altispira, Globigerinoides subquadratus, G. quadrilobatus (s.l.) (group), Globorotalia (T.) siakensis, G. (T.) scitula praescitula, Globigerina woodi woodi, G. woodi $\mathrm{cf}$. connecta, G. (T.) peripheroronda forma (of Blow, 1969).

Age: Zone N.6, Early Miocene. (see Note below).

Sample 3-15-9-6, 76-78 cm:

Rich planktonic foraminiferal fauna with Globigerina cf. foliata, $G$. cf. falconensis, $G$. woodi woodi, $G$. woodi connecta, G. praebulloides (s.l.), G. juvenilis, Globigerinoides subquadratus, G. quadrilobatus altiaperturus, G. quadrilobatus trilobus, G. quadrilobatus immaturus, Globigerinita dissimilis dissimilis, $G$. dissimilis ciperoensis, G. unicava unicava, G. incrusta, Globorotalia (T.) siakensis, G. (T.) scitula praescitula, Globoquadrina dehiscens dehiscens, G. cf. altispira (s.l.).

Age: Zone N.6, early Miocene. (see Note below).

Note concerning Cores 8 and 9, Hole 15: It will be seen from the records given above for the biostratigraphic records of Cores 8 and 9 , that Core 8 appears to be older than Core 9. Thus Core 8 is referable to the lower part of Zone N.6 and/or ? upper part of Zone N.5 whereas Core 9 is referable to Zone N.7 and upper part Zone N.6. If these cores were reversed in stratigraphic position there would be an essentially continuous stratigraphic record of horizons referable to Zones N.7 and N.6 (? also upper part Zone N.5). However, no misnumbering of the cores is likely and the explanation given by the shipboard scientists (teste letter dated 
26th May, 1969 from A. C. Pimm) is as follows: ..... "shipboard scientists attributed this to considerable movement of the ship during drilling. Thus Core 9 was taken during upward movement of the vessel and penetrated younger sediments above and to one side of the section that Core 8 sampled."

Hence the relative positions of Cores 8 and 9 should be considered as needing reversal when considering the actual sediments penetrated.

\section{Hole 16}

Sample 3-16-1-1, 77-79 cm:

Very rich planktonic foraminiferal fauna with Globorotalia (G.) hirsuta hirsuta, G. (G.) truncatulinoides truncatulinoides, $G$. (G.) truncatulinoides pachytheca, $G$. (T.) inflata, G. (T.) scitula scitula, Globigerinoides ruber, G. conglobatus conglobatus, G. quadrilobatus (group), Globigerina calida calida, Sphaeroidinella dehiscens dehiscens, G. oscitans, G. rubescens, Hastigerina pelagica, H. siphonifera siphonifera, Globigerina (Beella) digitata, etc.

Age: Zone N.23, Late Pleistocene/Holocene.

Sample 3-16-1-2, 76-78 cm:

As above.

Sample 3-16-1-4, 0-3 cm:

Rich planktonic foraminiferal fauna with Globorotalia (G.) cultrata menardii, $G$. (G.) hirsuta hirsuta, G. (G.) truncatulinoides truncatulinoides, $G$. (G.) crassula conomiozea, Globorotalia (Turborotalia) crassaformis crassaformis, G. (T.) inflata, G. (T.) scitula scitula, Globigerinoides ruber (red), G. conglobatus conglobatus, $G$. quadrilobatus (group), G. elongatus, G. elongatus pyramidalis, Globigerina falconensis, $G$. cf. bulbosa, $G$. calida praecalida, Hastigerina siphonifera siphonifera.

Age: Zone N.22, Pleistocene.

Sample 3-16-2-1, $150 \mathrm{~cm}$ :

As above.

Sample 3-16-2-2, $150 \mathrm{~cm}$ :

As above.

Sample 3-16-2-3, $150 \mathrm{~cm}$ :

Rich planktonic foraminiferal fauna with Globorotalia (G.) truncatulinoides truncatulinoides, $G$. (G.) crassula conomiozea, G. (G.) crassula crassula, G. (G.) cultrata menardii, G. (T.) crassaformis crassaformis, G. (T.) crassaformis ronda, $G$. (T.) tosaensis tenuitheca, $G$. (T.) inflata, G. (T.) cf. ventriosa, G. (T.) obesa, Sphaeroidinella dehiscens dehiscens, Globigerina falconensis, $G$. calida praecalida, G. bulbosa, $G$. cf. parabulloides, $G$. decoraperta, G. elongatus, G. elongatus pyramidalis, G. ruber (white), G. conglobatus conglobatus, G. quadrilobatus (group), Hastigerina siphonifera siphonifera, Orbulina spp.

Age: Zone N.22, earlier part, Pleistocene.
Sample 3-16-2-4, $150 \mathrm{~cm}$ :

Rich planktonic foraminiferal fauna with Globorotalia (G.) truncatulinoides truncatulinoides (phylogenetically primitive), G. (G.) crassula conomiozea, $G$. (G.) crassula crassula, G. (G.) cultrata menardii, G. (Turborotalia) crassaformis crassaformis, G. (T.) crassaformis ronda, G. (T.) inflata, G. (T.) tosaensis tenuitheca, G. (T.) acostaensis acostaensis, Globigerinoides elongatus, $G$. conglobatus conglobatus, G. quadrilobatus (group), Sphaeroidinella dehiscens dehiscens, Orbulina spp. Age: Zone N.22, basal part, earliest Pleistocene.

Sample 3-16-2-5, $150 \mathrm{~cm}$ :

As above, but a few specimens only of $G$. (G.) truncatulinoides truncatulinoides (phylogenetically primitive) and fairly common $G$. (Turborotalia) tosaensis tenuitheca.

Age: Zone N.22, basal part, earliest Pleistocene.

Sample 3-16-2-6, 72-79 cm:

Rich planktonic foraminiferal fauna with Globorotalia (Turborotalia) tosaensis (s.l.)(rare), G. (T.) crassaformis crassaformis, $G$. (T.) crassaformis ronda, $G$. (G.) crassula crassula, G. (G.) crassula conomiozea, G. (G.) crassula viola, $G$. (G.) hirsuta $\mathrm{cf}$. praehirsuta, G. (Turborotalia) inflata, G. (T.) acostaensis acostaensis, Globigerina calida praecalida, G. quadrilatera, G. falconensis, G. parabulloides, $G$. cf. bulbosa, Globigerinoides conglobatus conglobatus, G. ruber, G. elongatus, G. quadrilobatus (group), Candeina nitida nitida, Hastigerina siphonifera siphonifera, Orbulina spp., Sphaeroidinella dehiscens dehiscens.

Age: Zone N.21, late Pliocene.

Sample 3-16-3-1, $150 \mathrm{~cm}$ :

Rich planktonic foraminiferal fauna. As above but without specimens referable to $G$. (T.) tosaensis tosaensis. Age: Probably Zone N.21, late Pliocene.

Sample 3-16-3-2, $150 \mathrm{~cm}$ :

Rich planktonic foraminiferal fauna with Globoquadrina altispira altispira, G. altispira cf. globosa, Globigerina (Beella) digitata praedigitata, Globigerina falconensis, G. quadrilatera, G. praebulloides, G. calida praecalida, G. decoraperta, Globigerinoides elongatus, $G$. elongatus pyramidalis, G. cf. ruber, G. quadrilobatus (group), Globorotalia (G.) cultrata exilis, G. (G.) crassula crassula, G. (G.) crassula conomiozea, $G$. (G.) crassula viola, $G$. (T.) crassaformis crassaformis, G. (T.) crassaformis ronda, G. (T.) cf. subscitula, Sphaeroidinella dehiscens dehiscens, Hastigerina siphonifera siphonifera, Orbulina spp., Globorotalia (G.) hirsuta praehirsuta, $G$. (T.) ventriosa.

Age: Zone N.21, later Pliocene.

Sample 3-16-3-3, $150 \mathrm{~cm}$ :

As above but also with specimens referable to Globorotalia (G.) multicamerata and G. (G.) cultrata cultrata. Age: Zone N.21, later Pliocene. 
Sample 3-16-3-4, $150 \mathrm{~cm}$ :

As above.

Age: Zone N.21, later Pliocene.

Sample 3-16-3-5, $150 \mathrm{~cm}$ :

As above.

Age: Zone N.21, later Pliocene.

Sample 3-16-3-6, $150 \mathrm{~cm}$ :

Rich planktonic foraminiferal fauna with Sphaeroidinellopsis seminulina seminulina, Globoquadrina altispira altispira, Globoquadrina altispira globosa, Globigerina (Beela) digitata praedigitata, Globigerina falconensis, $G$. parabulloides, $G$. quadrilatera, $G$. decoraperta, $G$. cf. juvenilis, Globorotalia (G.) crassula crassula, G. (G.) crassula conomiozea, $G$. (G.) crassula viola, $G$. (G.) cultrata exilis, G. (G.) cf. miocenica (v. rare, immature specimens), Globorotalia (T.) crassaformis crassaformis, $G$. (T.) crassaformis ronda, G. (T.) inflata, Globigerinoides conglobatus conglobatus, $G$. elongatus, $G$. quadrilobatus (group), G. bollii, Globorotalia (G.) hirsuta praehirsuta, Hastigerina siphonifera siphonifera, Sphaeroidinella dehiscens dehiscens forma immatura. Age: within the interval $\left[\begin{array}{l}\text { Zone N.21 } \\ \text { Zone N.20 }\end{array}\right]$, but with derived older material, i.e. Pliocene with reworked Miocene.

Sample 3-16-4-1, 76-78 cm:

Rich planktonic foraminiferal fauna with Sphaeroidinellopsis subdehiscens paenedehiscens, S. subdehiscens subdehiscens, $S$. seminulina seminulina, Sphaeroidinella dehiscens dehiscens forma immatura, Globoquadrina altispira altispira, Globigerina falconensis, $G$. decoraperta, G. parabulloides, Globorotalia (G.) hirsuta praehirsuta, G. (G.) crassula crassula, G. (G.) crassula viola, $G$. (G.) crassula conomiozea, $G$. (T.) crassaformis crassaformis, $G$. (T.) crassaformis ronda, $G$. (T.) cf. obesa, $G$. (T.) puncticulata, G. (T.) subscitula, Globigerinoides conglobatus conglobatus, G. elongatus, G. bollii, Globigerina cf. pseudofoliata, G. bulbosa, Hastigerina siphonifera siphonifera, Orbulina spp., Globorotalia (G.) multicamerata.

Age: Within the interval $\left[\begin{array}{l}\text { Zone N.21 } \\ \text { Zone N.20 }\end{array}\right]$, but with derived older material, e.g., Pliocene with reworked Miocene.

Sample 3-16-4-2, 76-78 cm:

As above.

Age: Within the interval $\left[\begin{array}{l}\text { Zone N.21 } \\ \text { Zone N.20 }\end{array}\right]$, but with derived older material, e.g., Pliocene with reworked Miocene.

Sample 3-16-4-3, 72-74 cm:

Rich planktonic foraminiferal fauna with Globorotalia (G.) margaritae, G. (G.) crassula crassula, G. (G.) crassula conomiozea, G. (T.) crassaformis crassaformis, $G$.
(T.) crassaformis ronda, Globigerina decoraperta, G. bulbosa, G. falconensis, G. parabulloides, Globigerinoides elongatus, $G$. conglobatus conglobatus, $G$. quadrilobatus (group), G. bollii, Sphaeroidinella dehiscens dehiscens forma immatura, Sphaeroidinellopsis subdehiscens paenedehiscens, $S$. subdehiscens subdehiscens, $S$. seminulina seminulina, Globoquadrina altispira altispira.

Age: Probably within the interval $\left[\begin{array}{l}\text { Zone N.21 } \\ \text { Zone N.20 }\end{array}\right]$, but with derived older material, e.g., Pliocene with derived Miocene.

Sample 3-16-4-4, 72-74 cm:

Sphaeroidinella dehiscens dehiscens immatura, Sphaeroidinellopsis subdehiscens (s.l.), Globigerina decoraperta, G. bulbosa, G. falconensis, Globoquadrina altispira altispira, Globorotalia (G.) crassula crassula, G. (G.) crassula viola, G. (G.) crassula conomiozea, Globorotalia (T.) crassaformis crassaformis, G. (T.) crassaformis ronda, G. (T.) acostaensis humerosa (very rare), Globorotalia (G.) margaritae, Globigerinoides conglobatus conglobatus, G. elongatus, G. quadrilobatus (group). Age: Within the interval $\left[\begin{array}{l}\text { Zone N.21 } \\ \text { Zone N.20 }\end{array}\right]$, but with derived older material.

Sample 3-16-4-5, $77-79 \mathrm{~cm}$ :

As above.

Sample 3-16-4-6, $150 \mathrm{~cm}$ :

As above but with first occurrence of Globigerina nepenthes (3 specimens).

Age: Probably Zone N.19, Pliocene, mixed with older derived material.

Sample 3-16-5-1, $150 \mathrm{~cm}$ :

Globigerina nepenthes (rare), $G$. falconensis, $G$. decoraperta, $G$. bulbosa, $G$. cf. foliata, Globigerinita incrusta, Globorotalia (G.) miocenica (rare), G. (G.) cultrata exilis, G. (G.) multicamerata, G. (G.) crassula crassula, $G$. (G.) crassula conomiozea, $G$. (T.) crassaformis crassaformis, G. (G.) margaritae (some specimens phylogenetically very primitive), Globoquadrina altispira altispira, Sphaeroidinella dehiscens dehiscens forma immatura, Sphaeroidinellopsis subdehiscens (s.l.), S. seminulina seminulina.

Age: Probably Zone N.19, Pliocene, mixed with older derived material.

Sample 3-16-5-2, $150 \mathrm{~cm}$ :

Sample strongly contaminated with much recent Pleistocene Zone N.22/N.23 material. Sample unreliable.

Age: None.

Sample 3-16-5-3, $150 \mathrm{~cm}$ :

Same as for Sample 3-16-5-1, $150 \mathrm{~cm}$.

Age: Probably Zone N.19, Pliocene, mixed with older derived material. 
Sample 3-16-5-4, $150 \mathrm{~cm}$ :

Much comminuted planktonic foraminiferal fauna (?strong bioturbation). Globigerinoides elongatus, G. bollii, G. conglobatus conglobatus, G. quadrilobatus (group), Globoquadrina altispira altispira, G. altispira cf. globosa, Globigerina nepenthes, $G$. decoraperta, $G$. falconensis, G. parabulloides, Globorotalia (G.) crassula crassula, G. (G.) crassula conomiozea, G. (T.) crassaformis crassaformis, $G$. (T.) crassaformis ronda, $G$. (T.) subscitula, Sphaeroidinellopsis subdehiscens (s.l.), S. seminulina (s.l.).

Age: Within the interval $\left[\begin{array}{l}\text { Zone N.19 } \\ \text { Zone N.18 }\end{array}\right]$, Pliocene.

Sample 3-16-5-5, $150 \mathrm{~cm}$ :

As above.

Sample 3-16-5-6, $150 \mathrm{~cm}$ :

As above.

Sample 3-16-6-1, $150 \mathrm{~cm}$ :

Fairly rich, much comminuted planktonic foraminiferal fauna without much species diversification but with Echinoid spines. Globigerina nepenthes, G. decoraperta, Globorotalia (G.) miozea (s.l., probably immature specimens), G. (G.) crassula cf. conomiozea (v. rare), G. (T.) crassaformis crassaformis (v. rare), Globoquadrina altispira cf. altispira, Sphaeroidinellopsis subdehiscens (s.l.), S. seminulina (s.l.), Globigerinoides conglobatus conglobatus.

Age: Probably Zone N.18, earliest Pliocene or latest Miocene.

Sample 3-16-6-2, $150 \mathrm{~cm}$ :

Planktonic foraminiferal fauna without much species diversification. Globigerina nepenthes (abundant), Sphaeroidinellopsis subdehiscens (s.l.) (abundant), Globigerinoides conglobatus cf. conglobatus, G. conglobatus cf. canimarensis, Globorotalia (G.) miozea (s.l.), Orbulina spp., Globigerinoides bollii, G. quadrilobatus (group).

Age: Probably Zone N.18, earliest Pliocene or latest Miocene.

Sample 3-16-6-3, $150 \mathrm{~cm}$ :

Same as above plus 2 specimens Candeina nitida (s.l.). Age: As above.

Sample 3-16-6-4, $150 \mathrm{~cm}$ :

As above.

Sample 3-16-6-5, $150 \mathrm{~cm}$ :

As above.

Sample 3-16-6-6, $150 \mathrm{~cm}$ :

As above.

Sample 3-16-7-1, $150 \mathrm{~cm}$ :

Planktonic foraminiferal fauna with very little species diversification. As above.
Sample 3-16-7-2, $150 \mathrm{~cm}$ :

As above.

Sample 3-16-7-3, $150 \mathrm{~cm}$ :

As above.

Sample 3-16-7-4, $150 \mathrm{~cm}$ :

As above.

Sample 3-16-7-5, $150 \mathrm{~cm}$ :

As above.

Sample 3-16-7-6, $150 \mathrm{~cm}$ :

As above.

Sample 3-16-8-1, $150 \mathrm{~cm}$ :

As above but with some contamination from younger horizons.

Age: As above.

Sample 3-16-8-2, $150 \mathrm{~cm}$ :

Globigerina nepenthes, Globigerinoides conglobatus cf. conglobatus, G. conglobatus canimarensis, G. elongatus, G. bollii, Globorotalia (G.) crassula viola, G. (T.) crassaformis crassaformis, $G$. (T.) crassaformis ronda, Sphaeroidinellopsis subdehiscens (s.l.), G. (G.) miozea (s.l.), Orbulina spp.

Age: Within the interval $\left[\begin{array}{l}\text { Zone N.18 } \\ \text { Zone N.17 }\end{array}\right]$, Late Miocene.

Sample 3-16-8-3, $150 \mathrm{~cm}$ :

As above.

Sample 3-16-8-4, $150 \mathrm{~cm}$ :

As above.

Sample 3-16-8-5, $150 \mathrm{~cm}$ :

As above.

Sample 3-16-8-6, $150 \mathrm{~cm}$ :

As above.

Sample 3-16-9-1, $150 \mathrm{~cm}$ :

Very little species diversification; fauna with Globigerina nepenthes, Globigerina decoraperta, G. cf. falconensis, Globigerinoides conglobatus cf. conglobatus, G. elongatus, G. bollii, Globorotalia (G.) miozea (s.l.), Orbulina spp., Sphaeroidinellopsis subdehiscens (s.l.), S. seminulina (s.l.).

Age: Within the interval $\left[\begin{array}{l}\text { Zone N.18 } \\ \text { Zone N.17 }\end{array}\right]$, Late Miocene.

Sample 3-16-9-2, $150 \mathrm{~cm}$ :

As above.

Sample 3-16-9-3, $150 \mathrm{~cm}$ :

As above. 
Sample 3-16-9-4, $150 \mathrm{~cm}$ :

As above.

Sample 3-16-9-6, $150 \mathrm{~cm}$ :

Rich planktonic foraminiferal fauna with a very restricted diversification. Globigerina nepenthes, G. cf. decoraperta, Globigerinoides bollii, G. quadrilobatus immaturus, Orbulina spp., Sphaeroidinellopsis subdehiscens subdehiscens, $S$. seminulina seminulina.

Age: Probably Zone N.17, Late Miocene.

Sample 3-16-10-1, $150 \mathrm{~cm}$ :

Fauna unreliable, heavily contaminated from younger horizons.

Age: Indeterminate.

Sample 3-16-10-2, $150 \mathrm{~cm}$ :

Rich planktonic foraminiferal fauna without much species diversification but with some contamination from younger horizons. Probable in situ fauna as for Sample 3-16-9-6, $150 \mathrm{~cm}$.

Age: Probably Zone N.17, Late Miocene.

Sample 3-16-10-3, $150 \mathrm{~cm}$ :

Sample unreliable; fauna heavily contaminated from younger horizons.

Age: Indeterminate.

Sample 3-16-10-4, $150 \mathrm{~cm}$ :

Sample unreliable; fauna heavily contaminated from younger horizons.

Age: Indeterminate.

Sample 3-16-10-5, $150 \mathrm{~cm}$ :

Sample unreliable; fauna heavily contaminated from younger horizons.

Age: Indeterminate.

Sample 3-16-10-6, $150 \mathrm{~cm}$ :

Sample unreliable; fauna heavily contaminated from younger horizons.

Age: Indeterminate.

Sample 3-16-11-1, $150 \mathrm{~cm}$ :

Rich planktonic foraminiferal fauna with Globorotalia

(G.) cultrata cf. cultrata, $G$. (G.) cultrata limbata, $G$.

(G.) miozea (s.l.), G. (T.) subscitula, Globigerinoides bollii, Globigerina nepenthes, G. falconensis, Sphaeroidinellopsis subdehiscens (s.l.) etc. with younger material.

Age: Within the interval $\left[\begin{array}{l}\text { Zone N.17 } \\ \text { Zone N.16 }\end{array}\right]$, Late Miocene.

Sample 3-16-11-2, $150 \mathrm{~cm}$ :

Sample unreliable; fauna heavily contaminated from younger horizons.

Age: Indeterminate.
Sample 3-16-11-3, $150 \mathrm{~cm}$ :

Sample unreliable; fauna heavily contaminated from younger horizons.

Age: Indeterminate.

Sample 3-16-11-4, $150 \mathrm{~cm}$ :

Sample unreliable; fauna heavily contaminated from younger horizons.

Age: Indeterminate.

Sample 3-16-11-5, $150 \mathrm{~cm}$ :

Rich planktonic foraminiferal fauna with Globigerina bulloides apertura, $G$. nepenthes, $G$. falconensis, $G$. parabulloides, Globigerinoides bollii, G. quadrilobatus (group), Globorotalia (G.) cultrata limbata, G. (G.) cultrata cultrata, G. (G.) miozea (s.l.), Orbulina spp., Sphaeroidinellopsis subdehiscens subdehiscens, $S$. seminulina seminulina.

Age: Probably Zone N.16, Tortonian, Late Miocene.

Sample 3-16-11-6, $150 \mathrm{~cm}$ :

Rich planktonic foraminiferal fauna with Globigerina nepenthes, $G$. falconensis, Globigerinoides bollii, $G$. quadrilobatus (group), Globoquadrina dehiscens dehiscens, Globigerinita incrusta, G. ambitacrena, Globorotalia (G.) cultrata limbata, G. (G.) cultrata cultrata, Orbulina spp., Sphaeroidinellopsis subdehiscens subdehiscens.

Age: Probably within the interval of

$$
\left[\begin{array}{l}
\text { Zone N.16 } \\
\text { Zone N.15 upper part, }
\end{array}\right] \text {, Later Miocene. }
$$

Notes on the succession seen at Hole 16: It has been difficult to achieve a definite biostratigraphic subdivision of the succession penetrated at this hole due to the presence of contaminating material from Recent horizons in many of the deeper cores especially in Core 10. Furthermore, because of the probable marginal position of the hole with regard to the occurrence of fully tropical planktonic foraminiferal assemblages, there are many peculiarities of the faunas seen. Thus, many of the samples from the pre- later Pliocene cores show restricted faunal associations which make specific zonal determinations difficult. This is additionally complicated by some reworking and bioturbation of the sediment. Accordingly, in many cases it has been only possible to give the approximate biostratigraphic limits of the horizons of provenance of the various samples studied. Notwithstanding the difficulties outlined above it would appear that the succession penetrated did not extend to horizons significantly older than those included in the youngest Middle Miocene or the oldest Late Miocene.

\section{Hole 17}

Sample 3-17-1-1, 75-77 cm:

Rich planktonic foraminiferal fauna with Globorotalia (G.) truncatulinoides truncatulinoides, $G$. (G.) crassula 
crassula, G. (G.) crassula viola, G. (T.) inflata, G. (T.) crassaformis crassaformis, G. (T.) acostaensis humerosa, Globigerina eggeri eggeri, G. (Beella) digitata cf. digitata, Globigerinoides ruber, G. conglobatus conglobatus, $G$. pyramidalis, Hastigerina siphonifera siphonifera, Sphaeroidinella dehiscens dehiscens, Orbulina spp.

Age: Zone N.22/Zone N.23, Pleistocene/Holocene.

Sample 3-17-1-2, 75-77 cm:

As above with $G$. (G.) cultrata menardii.

Age: As above.

Sample 3-17-1-3, 75-77 cm:

As above.

Sample 3-17-1-4, 76-78 cm:

As above.

Sample 3-17-1-5, 76-78 cm:

As above.

Sample 3-17-2-1, 75-77 cm:

Strong preservational change to that seen in Core 1 . Rich planktonic foraminiferal fauna with Pulleniatina primalis (fairly advanced), Sphaeroidinellopsis seminulina seminulina, S. subdehiscens paenedehiscens, Globorotalia (G.) crassula crassula, G. (G.) crassula cf. viola, G. (G.) miozea (s.l.), G. (G.) cf. multicamerata, Globoquadrina altispira altispira, G. cf. dehiscens dehiscens, G. altispira cf. globosa, Globorotalia (Turborotalia) crassaformis crassaformis, Globigerinoides conglobatus conglobatus, $G$. conglobatus cf, canimarensis, G. obliquus extremus, Orbulina spp.

Age: Zone N.18 or uppermost Zone N.17, Late Miocene.

Sample 3-17-2-2, 75-77 cm:

As above.

Sample 3-17-2-3, 78-80 cm:

As above.

Sample 3-17-2-4, 75-77 cm:

Globoquadrina altispira altispira, G. altispira globosa, Sphaeroidinellopsis subdehiscens cf. paenedehiscens, $S$. subdehiscens subdehiscens, $S$. seminulina seminulina, Globorotalia (G.) miozea (s.l.), G. (G.) margaritae, G. (G.) crassula crassula, G. (G.) crassula cf. viola, $G$. (T.) crassaformis crassaformis, Globigerinoides conglobatus conglobatus, G. conglobatus cf. canimarensis, G. obliquus extremus, Orbulina spp.

Age: Zone N.18 or upper part Zone N.17, Late Miocene.

Sample 3-17-2-4, 75-77 cm:

As above.

Sample 3-17-2-6, 75-77 cm:

Globorotalia (G.)miozea (s.l.), G. (G.) cf. crassula (s.l.),
G. (G.) margaritae, Globoquadrina altispira altispira, Globigerinoides conglobatus cf. conglobatus, G. bollii, G. elongatus, Orbulina spp., Globigerina nepenthes, Sphaeroidinellopsis subdehiscens subdehiscens, S. subdehiscens paenedehiscens.

Age: Probably Zone N.17, upper part, Late Miocene.

\section{Hole 17A}

Sample 3-17A-1-1, 76-78 cm:

Fairly rich planktonic foraminiferal fauna with Globoquadrina dehiscens dehiscens, G. altispira altispira, $G$. subquadratus, G. quadrilobatus (group), Globoquadrina dehiscens advena, Globigerinita incrusta, Globorotalia (G.) miozea (s.l.).

Age: Probably Zone N.7, Early Miocene.

Sample 3-17A-1-2, 74-76 cm:

G. juvenilis, Globoquadrina dehiscens dehiscens, G. dehiscens advena, G. altispira altispira, Globigerinoides quadrilobatus (group), Globigerinita incrusta, Globorotalia (G.) miozea (group), Sphaeroidinellopsis cf. seminulina (s.l.).

Age: Probably Zone N.7, Early Miocene.

Sample 3-17A-1-3, $75-77 \mathrm{~cm}$ :

Globorotalia (G.) miozea, G. (T.) cf. siakensis, Globoquadrina dehiscens dehiscens, G. dehiscens advena, Globigerinita incrusta, G. ambitacrena, Globorotaloides hexagona variabilis, Sphaeroidinellopsis seminulina, $G$. falconensis, G. juvenilis, Globigerinoides quadrilobatus (group), Globigerinoides cf. subquadratus.

Age: Probably Zone N.7, Early Miocene.

Sample 3-17A-1-4, 75-77 cm:

Globorotalia (G.) miozea miozea, G. (T.) scitula cf. gigantea, G. quadrilobatus (group), Globoquadrina altispira altispira, G. altispira cf. globosa, G. dehiscens dehiscens, G. dehiscens advena, Globigerina woodi (s.l.), G. falconensis, Globorotaloides hexagona variabilis, Globigerinita incrusta, Sphaeroidinellopsis seminulina (s.s.), Globigerinoides subquadratus.

Age: Zone N.7, Early Miocene.

Sample 3-17A-1-5, 75-77 cm:

Poor planktonic foraminiferal fauna with Globigerina woodi woodi, G. woodi connecta, Globigerinoides quadrilobatus immaturus, G. quadrilobatus trilobus, Globigerinita unicava unicava.

Age: Probably Zone N.6, Aquitanian, Early Miocene.

Sample 3-17A-1-6, 78-80 cm:

Fair planktonic foraminiferal fauna Globorotalia (Turborotalia) sp. nov., Globigerinita incrusta, G. unicava unicava, G. dissimilis (s.l.), Globigerinoides quadrilobatus immaturus, G. quadrilobatus trilobus, Globoquadrina altispira cf. altispira, Globigerina woodi woodi, $G$. woodi connecta, G. bradyi, G. juvenilis.

Age: Probably Zone N.6 or late Zone N.5, Aquitanian, Early Miocene. 
Sample 3-17A-2-1, 71-73 cm:

Poor planktonic foraminiferal fauna with Globigerina woodi woodi, Globigerinoides quadrilobatus immaturus, G. quadrilobatus cf. trilobus, Globigerinita dissimilis ciperoensis, G. dissimilis dissimilis, G. unicava unicava. Age: Probably Zone N.5, Early Miocene.

Sample 3-17A-2-2, 75-77 cm:

As above.

Sample 3-17A-2-3, 75-77 cm:

Fair planktonic foraminiferal fauna with Globoquadrina dehiscens dehiscens, G. dehiscens praedehiscens, Globigerinoides quadrilobatus immaturus, $G$. cf. subquadratus, Globigerinita dissimilis (s.l.), G. unicava unicava, G. incrusta, Globigerina woodi woodi, Globorotalia (T.) siakensis.

Age: Zone N.5, Early Miocene, Aquitanian.

Sample 3-17A-2-4, 75-77 cm:

Globorotalia (T.) pseudokugleri, G. (T.) cf. kugleri, Globigerina juvenilis, G. bradyi, G. woodi (s.l.), G. praebulloides (s.l.), Globigerinita unicava unicava, G. dissimilis (s.l.), Globigerinoides quadrilobatus cf. immaturus, Globigerinita stainforthi praestainforthi, G. incrusta. Age: Zone N.4, Early Miocene.

Sample 3-17A-2-5, 75-77 cm:

As above.

Age: Zone N.4, Early Miocene.

Sample 3-17A-2-6, 75-77 cm:

Globorotalia (T.) kugleri, G. (T.) cf. pseudokugleri, G. (T.) siakensis, G. (T.) mendacis, Globigerina praebulloides (s.l), Globigerinita incrusta, G. dissimilis (s.l.), G. unicava unicava, Globoquadrina dehiscens praedehiscens, G. cf. altispira globularis, G. baroemoenensis, Cassigerinella chipolensis, Globorotaloidessuteri. (Note: No specimens referable to Globigerinoides spp.) Chiloguembelina sp. (2 specimens).

Age: Probably Zone N.3 uppermost part, but possibly early Zone N.4; latest Oligocene or possibly earliest Miocene.

Sample 3-17A-3-1, 75-77 cm:

Globigerina gortanii gortanii, G. tripartita, Globoquadrina $\mathrm{cf}$. dehiscens praedehiscens, G. prasaepis, Globorotalia (T.) opima opima, G. (T.) cf. siakensis (of Blow, 1969), G. (T.) permicra, G. (T.) clemenciae, Globigerinita dissimilis dissimilis, G. unicava unicava, Globorotaloides suteri, Chiloguembelina spp.

Age: Zone N.2, late Oligocene.

Sample 3-17A-3-6, 148-150 cm:

Globorotalia (T.) opima nana, forms ex interc $G$. (T.) opima nana and $G$. (T.) opima opima, $G$. (T.) siakensis, G. (T.) permicra, G. (T.) postcretacea, Globigerina cf. galavisi, forms ex interc G. galavisi and Globoquadrina altispira globularis, G. sellii, G. tripartita, G. winkleri,
G. gortanii gortanii, G. prasaepis, G. anguliofficinalis, Globigerinita dissimilis (s.l.), G. unicava unicava, G. unicava cf. primitiva, and very rare Globigerina $\mathrm{cf}$. ampliapertura, Chiloguembelina spp.

Age: Probably topmost part of Zone N.1 (=P.20).

Sample 3-17A-4-2, 73-75 cm:

Globigerina anguliofficinalis, G. ampliapertura, G. selli, G. tripartita, G. galavisi, G. cf. pseudovenezuelana, $G$. angiporoides, Globorotalia (T.) cf. increbescens, G. (T.) opima nana, G. (T.) postcretacea, G. (T.) permicra, Globigerinita unicava primitiva, G. martini scandretti, G. dissimilis (s.l.), Chiloguembelina sp.

Age: Probably Zone P.19, Oligocene. (See note below).

Sample 3-17A-4-5, 0-2 cm:

Globigerina tapuriensis, G. prasaepis, G. pseudovenezuelana, G. angiporoides, G. tripartita, G. galavisi, G. cf. sellii, Globorotalia (T.) increbescens, G. (T.) permicra, $G$. (T.) postcretacea, Globigerinita martini scandretti, G. unicava primitiva, G. dissimilis (s.l.), Globorotaloides suteri, Chiloguembelina spp.

Age: Probably Zone P.19, Oligocene. (See note below).

Sample 3-17A-4-6, 73-75 cm:

As above.

Note on Hole 17A: In this hole no specimens referable to Pseudohastigerina were seen. However, the occurrence of $G$. angiporoides, $G$. tapuriensis and $G$. pseudovenezuelana in samples from Core 4 suggests Zone P.19, middle part of the Oligocene.

\section{Hole 17B}

Sample 3-17B-1-1, 75-77 cm:

Globigerinita dissimilis dissimilis, G. dissimilis ciperoensis, G. unicava unicava, G. cf. incrusta, Globorotaloides suteri, Globigerina venezuelana, G. sellii, Globoquadrina dehiscens praedehiscens, Globorotalia (T.) mendacis, G. (T.) siakensis, G. (T.) clemenciae, G. (T.) gemma, G. (T.) permicra.

Age: Probably Zone'N.3, Oligocene.

Sample 3-17B-1-2, 76-78 cm:

Globigerina angulisuturalis (rare), G. juvenilis, G. praebulloides praebulloides, Globoquadrina dehiscens praedehiscens, Globigerinita dissimilis ciperoensis, G. unicava unicava, G. cf. incrusta, Globorotalia (T.) mendacis, G. (T.) cf. siakensis, G. (T.) gemma, G. (T.) permicra, G. (T.) clemenciae, Globigerina sellii.

Age: Probably Zone N.3, Oligocene.

Sample 3-17B-1-3, 76-78 cm:

As above.

Sample 3-17B-1-4, 75-77 cm:

Globigerina angulisuturalis (rare), G. sellii, G. prasaepis, $G$. praebulloides praebulloides, Globorotalia (T.) $\mathrm{cf}$. 
siakensis, G. (T.) cf. opima nana, G. (T.) mendacis, $G$. (T.) gemma, G. (T.) permicra, Globigerinita dissimilis dissimilis, G. unicava unicava, $G$. cf. incrusta.

Age: Probably Zone N.3, Oligocene.

Sample 3-17B-1-5, 75-76 cm:

G. cf. sellii, G. prasaepis, G. praebulloides (s.l.), Globorotalia (T.) mendacis, $G$. (T.) cf. opima nana, G. (T.) permicra, G. (T.) gemma, Globigerinita dissimilis (s.l.), $G$. unicava unicava, $G$. cf. incrusta, Globorotaloides suteri.

Age: Zone N.3 of Zone N.2, Oligocene.

Sample 3-17B-1-6, 73-75 cm:

As above.

Age: Zone N.3 or Zone N.2, Oligocene.

Sample 3-17B-2-1, 75-77 cm:

Globigerina angulisuturalis, G. prasaepis, G. sellii, G. juvenilis, Globorotalia (T.) cf. siakensis, G. (T.) gemma, $G$. (T.) permicra, Globorotaloides suteri, Globigerinita dissimilis (s.l.), G. unicava unicava, G. cf. pera, G. (T.) opima nana, G. (T.) opima cf. opima.

Age: Zone N.2 (=P.21), Oligocene.

Sample 3-17B-2-2, 76-78 cm:

As above.

Sample 3-17B-2-3, 75-77 cm:

As above.

Sample 3-17B-2-4, 76-78 cm:

Globigerina angulisuturalis (rare), $G$. cf. galavisi, $G$. sellii, G. cf. tripartita, G. juvenilis, Globorotalia (T.) cf. siakensis, G. (T.) opima nana, $G$. (T.) permicra, G. (T.) gemma, Globorotaloides suteri, Globigerinita dissimilis dissimilis, G. unicava unicava.

Age: Zone N.2 (=P.21), Oligocene.

Sample 3-17B-2-5, 75-77 cm:

Globigerina angulisuturalis (common), G. cf. galavisi, $G$. sellii, $G$. winkleri, $G$. prasaepis, $G$. anguliofficinalis, Globorotalia (T.) cf. siakensis, $G$. (T.) opima nana, $G$. (T.) gemma, G. (T.) permicra, Globigerinita unicava unicava, G. dissimilis (s.l.), G. (T.) opima cf. opima.

Age: Zone N.2 (=P.21), Oligocene.

Sample 3-17B-2-6, 75-77 cm:

Globigerina cf. ampliapertura, $G$. anguliofficinalis, ex interc $G$. angulisuturalis, G. prasaepis, G. cf. galavisi, Globorotalia (T.) cf. siakensis, $G$. (T.) opima nana, $G$. (T.) gemma, G. (T.) permicra, Globorotaloides suteri, Globigerinita unicava unicava, G. dissimilis dissimilis. Age: Early part Zone N.2 (=P.21) or later part Zone N.1 (=P.20), Oligocene.

Sample 3-17B-3-1, 76-78 cm:

As above.
Sample 3-17B-3-2, 75-77 cm:

Globigerina ampliapertura, G. prasaepis,.G. cf. galavisi, $G$. tripartita, $G$. winkleri, Globigerinita unicava unicava, G. dissimilis dissimilis, G. pera, Globorotalia (T.) gemma, G. (T.) permicra, G. (T.) opima nana, etc.

Age: Zone N.1 (=P.20), Oligocene.

Sample 3-17B-3-3, 75-77 cm:

Rich planktonic foraminiferal fauna with Globigerina ampliapertura, G. anguliofficinalis, G. angustiumbilicata, $G$. officinalis, $G$. juvenilis, $G$. prasaepis, $G$. tripartita, $G$. cf. galavisi, Globorotalia (T.) opima nana, G. (T.) opima cf. opima, G. (T.) gemma, G. (T.) permicra, G. (T.) clemenciae, Globigerinita dissimilis (s.s.), G. dissimilis ciperoensis, G. unicava unicava, Globorotaloides suteri, Cassigerinella chipolensis.

Age: Zone N.1 (=P.20), Oligocene.

Sample 3-17B-3-4, 75-77 cm:

As above.

Age: Zone N.1 (=P.20), Oligocene.

Sample 3-17B-3-5, 75-77 cm:

As above.

Age: Zone N.1 (=P.20), Oligocene.

Sample 3-17B-3-6, $75-77 \mathrm{~cm}$ :

As above.

Age: Zone N.1 (=P.20), Oligocene.

Sample 3-17B-4-1, 76-78 cm:

Globigerina ampliapertura, $G$. anguliofficinalis, $G$. angustiumbilicata, G. cf. juvenilis, G. prasaepis, G. tripartita, G. cf. galavisi, Globorotalia (T.) opima nana, G. (T.) opima cf. opima, G. (T.) gemma, G. (T.) clemenciae, G. (T.) permicra, Globigerinita unicava unicava, G. unicava cf. primitiva, G. dissimilis dissimilis, G. dissimilis ciperoensis, Globorotaloides suteri.

Age: Zone N.1 (=P.20), Oligocene.

Sample 3-17B-4-2, 75-77 cm:

As above plus Globigerinita martini scandretti.

Age: Zone N.1 (=P.20), earlier part, Oligocene.

Sample 3-17B-4-3, 76-78 cm:

Globigerina ampliapertura, G. anguliofficinalis, G. pseudovenezuelana, G. prasaepis, $G$. cf. sellii, G. tripartita, G. galavisi, Globigerinita martini scandretti, G. unicava primitiva, G. dissimilis dissimilis, G. cf. pera, Globorotalia (T.) cf. increbescens, G. (T.) opima nana, G. (T.) gemma, G. (T.) permicra, G. praebulloides occlusa, G. praebulloides leroyi.

Age: Probably Zone P.19, Oligocene (see note below).

Sample 3-17B-4-4, 74-76 cm:

As above, but with $G$. angiporoides.

Age: Zone P.19, Oligocene. 
Sample 3-17B-4-5, 75-77 cm:

As above.

Age: Zone P.19, Oligocene.

Sample 3-17B-4-6, 75-77 cm:

As above.

Age: Zone P.19, Oligocene.

Note on Hole 17B: As in Hole 17A, no specimens of Pseudohastigerina were seen in the planktonic foraminiferal assemblages of Core 4, Hole 17B. However, G. angiporoides and $G$. pseudovenezuelana occur and these species indicate the occurrence of Zone P.19. The absence of Pseudohastigerina in this part of the South Atlantic is extremely interesting and appears to be correlated with the extreme paucity of specimens referable to Cassigerinella also noted for Holes 17A and $17 \mathrm{~B}$.

\section{Hole 18}

Sample 3-18-1-5, 75-77 cm:

Rich planktonic foraminiferal fauna with Globorotalia (G.) truncatulinoides, G. (G.) crassula crassula, G. (G.) crassula viola, G. (G.) cultrata cultrata, $G$. (G.) cultrata menardii, Globorotalia (Turborotalia) inflata, G. (T.) crassaformis ronda, G. (T.) crassaformis (s.s.), Globigerinoides conglobatus conglobatus, $G$. elongatus, $G$. pyramidalis, G. cf. ruber, G. quadrilobatus (group), Sphaeroidinella dehiscens dehiscens.

Age: Zone N.22, Pleistocene.

Sample 3-18-2-1, 0-2 cm:

Sphaeroidinellopsis cf. seminulina (s.s.), Globigerinita dissimilis (s.s.), G. stainforthi (s.s.) (rare), G. incrusta, $G$. ambitacrena, Globigerinatella cf. insueta (v. rare), Globorotalia (T.) peripheroronda, G. (T.) praescitula, Globigerinoides subquadratus, G. quadrilobatus (group), Globoquadrina dehiscens (s.s.), G. altispira (group). Age: Zone N.6, Early Miocene.

Sample 3-18-2-2, 75-77 cm:

Globigerinatella $\mathrm{cf}$. insueta (phylogenetically very primitive), Globigerinita dissimilis (s.s.), G. unicava unicava, G. incrusta, G. ambitacrena, Globorotaloides cf. suteri, Globigerinoides subquadratus, G. quadrilobatus (group), Globoquadrina altispira altispira, G. altispira globosa, $G$. dehiscens dehiscens, $G$. dehiscens praedehiscens, Globorotalia (T.) peripheroronda, G. (T.) siakensis, G. (T.) n. sp.

Age: Zone N.6, probably early part, Early Miocene.

Sample 3-18-2-3, 148-150 cm:

As above.

Sample 3-18-2-4, 148-150 cm:

Globigerinita dissimilis(s.s.), G. unicava (s.s.), G. incrusta, G. ambitacrena, Globorotalia (T.) siakensis, G. (T.) peripheroronda, Globoquadrina dehiscens (s.s.), G. dehiscens praedehiscens, Globigerina woodi woodi, $G$. woodi connecta, Globigerinoides quadrilobatus (s.l.), Globorotaloides cf. suteri.

Age: Prøbably upper part Zone N.5, Early Miocene.

Sample 3-18-2-5, 148-150 cm:

As above.

Age: Zone N.5, Early Miocene.

Sample 3-18-2-6, sample interval not given:

As above.

Age: Zone N.5, Early Miocene.

Sample 3-18-3-1, 148-150 cm:

As above.

Sample 3-18-3-2, 75-77 cm:

As above.

Age: Zone N.5, Early Miocene.

Sample 3-18-3-3, 148-150 cm:

As above.

Age: Zone N.5, Early Miocene.

Sample 3-18-3-4, 148-150 cm:

As above.

Age: Zone N.5, Early Miocene.

Sample 3-18-3-5, 148-150 cm:

As above.

Age: Zone N.5, Early Miocene.

Sample 3-18-3-6, 148-150 cm:

Globigerinita dissimilis (s.s.), G. unicava (s.s.), G. incrusta, G. ambitacrena, Globigerina praebulloides (s.l.), $G$. woodi woodi, $G$. woodi connecta, Globigerinoides quadrilobatus immaturus (rare), Globoquadrina dehiscens praedehiscens, Globigerina juvenilis, G. bradyi. Age: Either lower Zone N.5 or upper Zone N.4, Early Miocene.

Sample 3-18-4-2, 0-2 cm:

Globigerinita dissimilis (s.l.), G. unicava unicava, G. incrusta, G. ambitacrena, Globigerina juvenilis, G. bradyi, $G$. cf. woodi woodi, G. praebulloides (s.l.), Globigerinoides quadrilobatus immaturus (rare), Globorotalia (T.) kugleri, G. (T.) siakensis, Globoquadrina baroemoenensis, $G$. dehiscens praedehiscens, Globigerina cf. winkleri, $G$. cf. binaiensis (rare).

Age: Zone N.4, Early Miocene.

Sample 3-18-4-3, 0-2 cm:

As above, but no specimens referable to Globigerinoides (s.l.).

Age: Zone N.4, Early Miocene (from the evidence of next deeper sample).

Sample 3-18-4-4, 75-77 cm:

Globigerinoides quadrilobatus primordius, Globigerina 
praebulloides occlusa, G. juvenilis, $G$. woodi woodi, $G$. venezuelana, Globoquadrina dehiscens praedehiscens, G. baroemoenensis, Globigerinita dissimilis, G. unicava unicava, Globorotaloides suteri, Globorotalia (T.) kugleri, G. (T.) pseudokugleri, G. (T.) siakensis.

Age: Zone N.4, Early Miocene.

Sample 3-18-4-5, $148-150 \mathrm{~cm}$ :

As above.

Age: Zone N.4, Early Miocene.

Sample 3-18-5-1, 148-150 cm:

As above.

Age: Zone N.4, Early Miocene.

Sample 3-18-5-2, 75-77 cm:

As above.

Age: Zone N.4, Early Miocene.

Sample 3-18-5-3, 148-150 cm:

As above.

Age: Zone N.4, Early Miocene.

Sample 3-18-5-6, 0-2 cm:

As above.

Age: Zone N.4, Early Miocene.

Sample 3-18-6-1, 74-76 cm:

As above, but no specimens of Globigerinoides quadrilobatus primordius notwithstanding abundant occurrence of Globigerina praebulloides occlusa. Sample also contains rare $G$. ouachitaensis ciperoensis f. atypica (of Blow, 1969) and G. mendacis.

Age: Zone N.3, upper part, Late Oligocene.

Sample 3-18-6-2, 148-150 cm:

As above.

Age: Zone N.3, upper part, Late Oligocene.

Sample 3-18-6-3, 148-150 cm:

Globorotalia (T.) mendacis, G. (T.) cf. pseudokugleri, $G$. (T.) cf. kugleri, Globigerina angustiumbilicata, G. ouachitaensis ciperoensis f. atypica (of Blow, 1969), $G$. cf. sellii, $G$. venezuelana, Globoquadrina dehiscens praedehiscens, Globigerina praebulloides occlusa, $G$. cf. enapertura, G. woodi(s.l.), Globigerinita dissimilis (s.l.), $G$. unicava unicava.

Age: Zone N.3, upper part, Late Oligocene.

Sample 3-18-6-4, 148-150 cm:

As above.

Age: Zone N.3, upper part, Late Oligocene.

Sample 3-18-6-6, 73-75 cm:

As above.

Age: Zone N.3, upper part, Late Oligocene.

\section{Hole 19}

Sample 3-19-1-1, 75-77 cm:

No stratigraphically significant planktonic foraminifera. Age: None.

Sample 3-19-3-1, 73-75 cm:

Very poor planktonic foraminiferal fauna. Globigerinita dissimilis (s.l.), G. unicava (s.l.), Globigerina praebulloides occlusa, G. prasaepis.

Age: Within the interval $\left[\begin{array}{l}\text { Zone N.2 } \\ \text { Zone P.18 }\end{array}\right]$, Oligocene.

Sample 3-19-3-2, $77-79 \mathrm{~cm}$ :

As above.

Sample 3-19-3-3, 78-80 cm:

As above.

Sample 3-19-3-4, $72-74 \mathrm{~cm}$ :

As above.

Sample 3-19-3-5, 73-75 cm:

No significant planktonic foraminifera.

Age: None.

Sample 3-19-3-6, 75-77 cm:

As above.

Sample 3-19-4-1, 148-150 cm:

As above.

Sample 3-19-4-2, 73-75 cm:

Poor planktonic foraminiferal fauna with Globorotaloides suteri, Globigerinita unicava primitiva.

Age: Probably Oligocene.

Sample 3-19-4-3, 148-150 cm:

As above.

Sample 3-19-4-4, 148-150 cm:

Fair planktonic foraminiferal fauna with Globigerina angiporoides, G. prasaepis, G. cf. tapuriensis, Globigerinita unicava primitiva, G. dissimilis (s.l.), Globorotaloides suteri, Globorotalia (T.) opima nana.

Age: Within the interval $\left[\begin{array}{l}\text { Zone P.19 } \\ \text { Zone P.18 }\end{array}\right]$, Oligocene.

Sample 3-19-4-5, 148-150 cm:

As above.

Sample 3-19-4-6, 148-150 cm:

As above.

Sample 3-19-5-1, 148-150 cm:

No significant planktonic foraminifera.

Age: None.

Sample 3-19-5-2, 77-79 cm:

As above. 
Sample 3-19-5-3, 73-75 cm:

As above.

Sample 3-19-5-4, 77-79 cm:

Very poor planktonic foraminiferal fauna Globigerapsis index, Globigerinita dissimilis, G. unicava primitiva. Age: Late Eocene/basal Oligocene i.e. within interval Zone P.18 (basal part) Zone P.16

Sample 3-19-5-5, 73-75 cm:

No significant planktonic foraminifera.

Age: None.

Sample 3-19-5-6, 73-75 cm:

Same as for Sample 3-19-5-4, 77-79 cm.

Age: Late Eocene/basal Oligocene i.e. within interval $\left[\begin{array}{l}\text { Zone P.18 (basal part) } \\ \text { Zone P.16 }\end{array}\right]$.

Sample 3-19-6-1, 148-150 cm:

As above.

Sample 3-19-6-2, 148-150 cm:

Very poor planktonic foraminiferal fauna, Globigerapsis index only recognizable.

Age: As above.

Sample 3-19-6-3, 148-150 cm:

No significant planktonic foraminifera.

Age: None.

Sample 3-19-6-4, 148-150 cm:

As above.

Sample 3-19-6-5, 148-150 cm:

As above.

Sample 3-19-6-6, 148-150 cm:

Very poor planktonic foraminiferal fauna with Globigerapsis index only recognizable.

Age: Within interval $\left[\begin{array}{l}\text { Zone P.18 (basal) } \\ \text { Zone P.16 }\end{array}\right]$, Late Eocene/ basal Oligocene.

Sample 3-19-7-1, 73-75 cm:

As above.

Sample 3-19-7-2, 73-75 cm:

As above.

Sample 3-19-7-3, 73-75 cm:

As above.

Sample 3-19-7-4, 73-75 cm:

As above.

Sample 3-19-7-5, 73-77 cm:

Poor planktonie foraminiferal fauna with Globigerina senni and Globigerapsis index only recognizable.

Age: Late Eocene, within interval $\left[\begin{array}{l}\text { Zone P.16 } \\ \text { Zone P.14 }\end{array}\right]$

Sample 3-19-8-1, 148-150 cm:

Poor planktonic foraminiferal fauna with Globigerina senni, Acarinina spp., Truncorotuloides cf. rohri, Globigerapsis index.

Age: Middle Eocene, within interval $\left[\begin{array}{l}\text { Zone P.14 } \\ \text { Zone P.10 }\end{array}\right]$

Sample 3-19-8-2, 73-75 cm:

As above.

Sample 3-19-8-3, 148-150 cm:

As above.

Sample 3-19-8-4, 148-150 cm:

As above.

Sample 3-19-8-5, 148-150 cm:

As above.

Sample 3-19-8-6, 148-150 cm:

Acarina cf. densa, A. primitiva, Truncorotaloides spp., Globigerina senni, Globigerapsis index, Globigerina inaequispira (group), Globigerapsis kugleri.

Age: Within interval $\left[\begin{array}{l}\text { Zone P.12 } \\ \text { Zone P.11 }\end{array}\right]$, lower part Middle Eocene.

Sample 3-19-9-1, 148-150 cm:

As above.

Sample 3-19-9-2, 148-150 cm:

As above.

Sample 3-19-9-3, 148-150 cm:

As above.

Sample 3-19-9-4, 148-150 cm:

As above.

Sample 3-19-9-5, 148-150 cm:

As above.

Sample 3-19-9-6, 148-150 cm:

Very rich planktonic foraminiferal fauna. Globorotalia (Morozovella) aragonensis, Acarinina densa, Acarinina spp., G. (M.) cf. querra, Globigerapsis index, G. kugleri, Subbotina inaequispira, S. (?) cf. frontosa, S. linaperta, Globigerinoides (?) higginsi.

Age: Zone P.11, earlier Middle Eocene.

Sample 3-19-10-1, 148-150 cm:

As above.

Age: Zone P.11, earlier Middle Eocene.

Sample 3-19-10-2, 148-150 cm:

As above. 
Sample 3-19-10-3, 148-150 cm:

As above.

Sample 3-19-10-5, 148-150 cm:

As above.

Sample 3-19-10-6, 148-150 cm:

Rather poor fauna but with some taxa as above.

Age: Zone P.11, earlier Middle Eocene.

Sample 3-19-11-1, 148-150 cm:

Same as for Sample 3-19-10-5, 148-150 cm.

Age: As above.

Sample 3-19-11-2, 148-150 cm:

As above.

Sample 3-19-11-3, 148-150 cm:

As above.

Sample 3-19-11-4, 148-150 cm:

As above.

\section{Hole 20A}

Sample 3-20A-2-1, 73-75 cm:

Very poor planktonic foraminiferal fauna with Globigerinita unicava primitiva, G. dissimilis (s.l.), G. prasaepis, Globorotaloides suteri.

Age: Probably within the interval

$$
\left[\begin{array}{l}
\text { Zone N.1 (early part) } \\
\text { Zone P.18 }
\end{array}\right] \text {, Oligocene. }
$$

Sample 3-20A-2-2, 75-77 cm:

As above.

Sample 3-20A-2-3, 75-77 cm:

As above.

Sample 3-20A-2-4, 148-150 cm:

As above.

Sample 3-20A-2-5, 148-150 cm:

As above.

Sample 3-20A-2-6, 148-150 cm:

As above.

\section{Hole 20B}

Sample 3-20B-1-1, $73-75 \mathrm{~cm}$ :

Rather poor planktonic foraminiferal fauna with Globigerinita unicava primitiva, G. dissimilis (s.l.), Globigerina prasaepis, G. cf. galavisi.

Age: Within interval $\left[\begin{array}{l}\text { Zone N.1 (early part) } \\ \text { Zone P.18 }\end{array}\right]$

Sample 3-20B-1-2, 73-75 cm:

As above.
Sample 3-20B-1-3, 73-75 cm:

As above.

Sample 3-20B-1-4, $73-75 \mathrm{~cm}$ :

As above.

Sample 3-20B-1-5, 70-72 cm:

As above.

Sample 3-20B-1-6, 73-74 cm:

As above.

Hole 20C

Sample 3-20C-1-1, $72-74 \mathrm{~cm}$ :

No significant planktonic foraminifera.

Age: None.

Sample 3-20C-1-2, 76-78 cm:

As above.

Sample 3-20C-1-3, 71-73 cm:

As above.

Sample 3-20C-1-4, $72-74 \mathrm{~cm}$ :

As above.

Sample 3-20C-1-5, $71-73 \mathrm{~cm}$ :

As above.

Sample 3-20C-1-6, 76-78 cm:

As above.

Sample 3-20C-2-1, 148-150 cm:

Globigerina angiporoides, G. pseudovenezuelana, $G$. prasaepis, G. cf. tapuriensis, G. galavisi, Globigerinita unicava primitiva.

Age: Within the interval $\left[\begin{array}{l}\text { Zone P.19 } \\ \text { Zone P.18 }\end{array}\right]$, Oligocene.

Sample 3-20C-2-2, 148-150 cm:

As above.

Sample 3-20C-2-3, 71-73 cm:

As above.

Sample 3-20C-2-4, 74-76 cm:

As above.

Sample 3-20C-2-5, 148-150 cm:

No planktonic foraminifera present.

Age: Indeterminate.

Sample 3-20C-2-6, 148-150 cm:

Globigerina angiporoides, G. pseudovenezuelana, $G$. prasaepis, G. galavisi, G. tripartita, Globorotalia (T.) opima nana, G. (T.) increbescens, Globigerinita unicava primitiva, G. dissimilis (s.l.), Globorotaloides suteri. Age: Within the interval $\left[\begin{array}{l}\text { Zone P.19 } \\ \text { Zone P.18 }\end{array}\right]$, Oligocene. 
Sample 3-20C-3-1, 77-79 cm:

No significant planktonic foraminifera present.

Age: Indeterminate.

Sample 3-20C-3-2, 77-79 cm:

As above.

Sample 3-20C-3-3, $72-74 \mathrm{~cm}$ :

As above.

Sample 3-20C-3-4, 71-73 cm:

Globigerina sp., Globigerapsis index.

Age: Within the interval $\left[\begin{array}{l}\text { Zone P.18, early } \\ \text { Zone P.16 }\end{array}\right]$, Late Eocene/basal Oligocene.

Sample 3-20C-3-5, 77-79 cm:

As above.

Sample 3-20C-3-6, 76-78 cm:

No significant planktonic foraminifera present.

Age: Indeterminate.

Sample 3-20C-4-1, 76-78 cm:

As above.

Sample 3-20C-4-2, 76-78 cm:

Globigerapsis index, Globigerina senni, Acarinina spp. Age: Within the interval $\left[\begin{array}{l}\text { Zone P.14 } \\ \text { Zone P.10 }\end{array}\right]$, middle Eocene.

Sample 3-20C-4-3, 76-78 cm:

As above.

Sample 3-20C-4-4, 148-150 cm:

As above.

Sample 3-20C-5-1, 148-150 cm:

Globorotalia (Morozovella) aragonensis, Acarinina densa, Acarinina spp., A. cf. primitiva, Globigerina senni, $G$. inaequispira (group).

Age: Within interval $\left[\begin{array}{l}\text { Zone P.11 } \\ \text { Zone P.9 }\end{array}\right]$, lower part of Middle

Eocene to upper part of Lower Eocene.

Sample 3-20C-5-2, 75-77 cm:

As above.

Sample 3-20C-5-3, 78-80 cm:

As above.

Sample 3-20C-5-4, 77-79 cm:

Rich planktonic foraminiferal fauna. Globorotalia (Morozovella) aragonensis, G. (M.) lensiformis, G. (M.) cf. "spinulosa", Acarinina densa, A. soldadoensis angulosa, A. aspensis, $A$. primitiva, A. broedermanni, Subbotina inaequispira (group).

Age: Zone P.9, latest Lower Eocene.
Sample 3-20C-5-5, $72-74 \mathrm{~cm}$ :

As above.

Age: Zone P.9, latest Lower Eocene.

Sample 3-20C-5-6, 76-78 cm:

Globorotalia (Morozovella) subbotinae, G. (M.) marginodentata, G. (M.) formosa gracilis, G. (M.) quertra, G. (M.) aequa, Acarinina soldadoensis (s.s.), A. soldadoensis angulosa, G. (M.) aragonensis (rare), G. (M.) crosswickiensis.

Age: Zone P.8, Lower Eocene.

Sample 3-20C-6-1, 148-150 cm:

As above.

Age: Zone P.8, Lower Eocene.

Sample 3-20C-6-2, $72-74 \mathrm{~cm}$ :

Globorotalia (Morozovella) velascoensis (v. rare), G. (M.) crosswickiensis, G. (M.) subbotinae, G. (M.) formosa gracilis, G. (M.) marginodentata, Acarinina soldadoensis, A. soldadoensis angulosa, A. cf. broedermanni. Age: Either upper Zone P.6 or lower Zone P.7-i.e. close to the Lower Eocene/Paleocene boundary.

Sample 3-20C-6-3, 76-78 cm:

As above, but with $G$. (M.) velascoensis acuta.

Age: Probably Zone P.6, upper Paleocene.

Sample 3-20C-6-4, 72-74 cm:

Globorotalia (T.) uncinata, Globigerina spiralis, G. (T.) cf. imitata, G. (T.) compressa, G. (T.) pseudobulloides, (also some Globotruncana spp.).

Age: Zone P.2 with ?bioturbation of Maestrichtian.

Sample 3-20C-6-5, 81-83 cm:

Age: Late Maestrichtian.

\section{Hole 21}

Sample 3-21-1-1, 148-150 cm:

Sphaeroidinella dehiscens dehiscens, Sphaeroidinellopsis subdehiscens paenedehiscens, S. seminulina, Globigerina bulloides apertura, G. parabulloides, G. falconensis, G. bulbosa, G. decoraperta, Globigerinoides conglobatus conglobatus, G. elongatus, G. cf. ruber, G. quadrilobatus (group), Hastigerina siphonifera siphonifera, Globorotalia (G.) miocenica, $G$. (G.) cultrata exilis, $G$. (G.) multicamerata, $G$. (G.) crassula viola, $G$. (G.) crassula conomiozea, G. (Turborotalia) crassaformis crassaformis, G. (T.) crassaformis ronda, Globoquadrina altispira altispira, G. altispira globosa.

Age: Within the interval $\left[\begin{array}{l}\text { Zone N.20 } \\ \text { Zone N.19 }\end{array}\right]$, Pliocene.

Sample 3-21-1-2, 148-150 cm:

As above. 
Sample 3-21-1-3, 148-150 cm:

As above.

Sample 3-21-1-4, $87-89 \mathrm{~cm}$ :

Candeina nitida nitida, Globigerina bulbosa, G. parabulloides, $G$. falconensis, $G$. nepenthes (rare), G. decoraperta, Globorotalia (G.) miocenica, G. cf. cultrata exilis, G. multicamerata, G. (G.) miozea ciboaensis, $G$. (G.) crassula conomiozea, $G$. (G.) crassula viola, $G$. (Turborotalia) crassaformis crassaformis, G. (T.) crassaformis ronda, Globigerinoides conglobatus conglobatus, G. pyramidalis, G. elongatus, G. quadrilobatus (group), Globoquadrina altispira altispira, G. altispira globosa, Sphaeroidinella dehiscens forma immatura.

Age: Zone N.19, Zanclian, early Pliocene.

Sample 3-21-1-5, 148-150 cm:

As above.

Sample 3-21-1-6, 148-150 cm:

Sphaeroidinella dehiscens dehiscens forma immatura, Sphaeroidinellopsis subdehiscens paenedehiscens, $S$. seminulina seminulina, $S$. seminulina kochi, Candeina nitida nitida, Hastigerina siphonifera siphonifera, Globorotalia (G.) miocenica, $G$. (G.) multicamerata, G. (G.) cultrata exilis, G. (G.) miozea ciboaensis, $G$. (G.) crassula crassula, $G$. (G.) crassula conomiozea, G. (Turborotalia) crassaformis crassaformis, $G$. (T.) crassaformis ronda, Globoquadrina altispira altispira, G. altispira globosa, Globigerinoides conglobatus conglobatus, $G$. pyramidalis, G. elongatus, G. quadrilobatus (group). Age: Zone N.19, Zanclian, early Pliocene.

Sample 3-21-2-1, 148-150 cm:

Rich planktonic foraminiferal fauna, only the in situ fauna listed and Pliocene forms neglected. Globorotalia (Morozovella) subbotinae, G. (M.) cf. aequa, G. (M.) formosa gracilis, Globigerina (?) soldadrensis, A. convexa (group), Subbotina tritoculinoides (group), $S$. cf. linaperta, S. triangularis, Globorotalia (Turborotalia) chapmanni, G. (T.) reissi, Globorotalia (G.) cf. pseudoscitula elongata, $G$. (Morozovella) cf. crosswichiensis, Subbotina (?) primitiva, Subbotina (?) gravelli.

Age: Zone P.6, Londinian, Late Paleocene.

Sample 3-21-2-2, 0-2 cm:

Same as above, but also with $G$. (Morozovella) acuta. Age: Zone P.6, Londinian, Late Paleocene.

Sample 3-21-2-3, 148-150 cm:

Mixed planktonic foraminiferal assemblage. Globorotalia (G.) pseudomenardii, G. (T.) ehrenbergi, G. (Morozovella) velascoensis (s.s.), G. (M.) crosswichiensis, $G$. (M.) aequa, G. (M.) subbotinae, G. (M.) formosa gracilis, G. (Acarinina) convexa, G. (A.) cf. chascanona, Acarinina soldadoensis, $A$. cf. gravelli, G. (?) mckannai, Subbotina triloculinoides, $S$. triangularis, $S$. velascoensis (cf. Bolli), G. (T.) pseudobulloides, G. (T.) chapmanni.
Age: Zone P.6 mixed with Zone P.4 (i.e. Late Paleocene and middle Paleocene mixed).

Sample 3-21-2-4, 74-76 cm:

As above.

Age: Zone P.6 mixed with Zone P.4 (i.e. Late Paleocene and middle Paleocene mixed).

Sample 3-21-2-5, 83-85 cm:

Globorotalia (G.) pseudomenardii (rare), G. (Morozovella) velascoensis, $G$. (M.) aequa (common), G. (M.) crosswichiensis, G. (M.) cf. acutispira, G. (Turborotalia) reissi, G. (T.) cf. ehrenbergi, Acarinina (?) aquiensis, $A$. (?) chascanona, A. (?) tribulosa, A. (?) mckannai, Subbotina triloculinoides, $S$. velascoensis (of Bolli, 1957), $S$. triangularis.

Age: Probably upper part Zone P.4, middle Paleocene (Thanetian).

Sample 3-21-2-6, 73-75 cm:

Globorotalia (Morozovella) cf. velascoensis, G. (M.) crosswichiensis, G. (M.) cf. acutispira, G. (M.) aequa, G. (Turborotalia) ehrenbergi, G. (T.) cf. chapmanni, $G$. (Acarinina (?)) convexa, Globorotalia (G.) pseudomenardii, Acarinina (?) mckannai, $A$. (?) cf. tribulosa, $A$. (?) cf. aquiensis, Subbotina triloculinoides, S. triangularis, S. cf. velascoensis (of Bolli, 1957), G. (M.) angulata.

Age: Zone P.4, middle Paleocene (Thanetian).

Sample 3-21-3-1, 148-150 cm:

Mixed fauna (? mechanical mixing of core) containing specimens referable to Zones P.4 P.3, P.2 and P.1 with specimens referable to Late Maestrichtian.

Age: Horizon of penetration referable to Zone M.14, Late Maestrichtian.

Note on Hole 21: Samples received from Cores 3, 4, 5 , 6, 7 and 8 show a succession from Late Maestrichtian to early Campanian or possibly late Santonian.

\section{Hole 21A}

Sample 3-21A-1-1, 148-150 cm:

Hantkenina dumblei, Globigerapsis index, G. kugleri, Globigerapsis(?) higginsi, Globorotalia (Acarinina) densa, G. (A.) broedermanni, Subbotina linaperta, S. inaequispira (group), Globorotalia (Turborotalia) sp.. Globorotalia (Morozovella) sp. ex interc crassatalehneri, Pseudohastigerina micra, G. (Truncorotaloides) mayoensis, G. (T.) cf. topilensis, Subbotina ? frontosa (=boweri).

Age: Zone P.11, Lutetian, middle Eocene.

Sample 3-21A-1-2, 148-150 cm:

As above.

Age: Zone P.11, Lutetian, Middle Eocene. 
Sample 3-21A-1-3, 148-150 cm:

Globorotalia (G.) pseudoscitula, G. (Morozovella) crassata (= spinulosa of Bolli), G. (Acarinina) densa, G. (A.) broedermanni, Globigerina (?) frontosa, Globigerapsis (?) higginsi, G. kugleri, G. index, Globigerina senni, Subbotina linaperta, G. inaequispira, Pseudohastigerina micra, Globorotalia (Truncorotaloides) mayoensis, G. (T.) cf. topilensis.

Age: Zone P.11, Lutetian, middle Eocene.

Sample 3-21A-1-4, 148-150 cm:

Same as above but without Globigerapsis kugleri and Hantkenina dumblei.

Age: Zone P.10 or Zone P.11, early Lutetian, Middle Eocene.

Sample 3-21A-1-5, 148-150 cm:

Samples much contaminated with Pliocene to Recent material, but in situ fauna appears similar to that of Sample 3-21A-1-1, 148-150 cm noted above.

Age: Zone P.10 or Zone P.11, Lutetian, Middle Eocene.

Sample 3-21A-1-6, 148-150 cm:

As above.

Sample 3-21A-2-1, 148-150 cm:

Globorotalia (Morozovella) aragonensis, G. (M.) aragonensis caucasica, G. (M.) lensiformis, G. (M.) formosa cf. formosa, Globorotalia (Acarinina) primitiva, G. (A.) densa, G. (A.) cf. aspensis, G. (A.) broedermanni, Subbotina turgida, $S$. (?) taroubaensis, S. eocaena, S. inaequispira (group), $S$. linaperta, Pseudohastigerina micra, P. cf. wilcoxensis, Pararotalia cf. palmerae, Globorotalia (Acarinina) soldadoensis angulosa.

Age: Zone P.8 (Subzone P.8b), Cuisian, Lower Eocene.

Sample 3-21A-2-2, 148-150 cm:

As above.

Age: Zone P.8 (Subzone P.8b), Cuisian, Lower Eocene.

Sample 3-21A-2-3, (no sample position in section given): Sample heavily contaminated by Pleistocene/Pliocene/ Miocene, but in situ fauna probably same as above.

Age: ?Zone P.8, Cuisian, Lower Eocene.

Sample 3-21A-2-5, 148-150 cm:

As above.

Age: ?Zone P.8, Cuisian, Lower Eocene.

Sample 3-21A-2-6, 148-150 cm:

In situ fauna include Globorotalia (Morozovella) aragonensis (s.s.), G. (M.) aragonensis caucasica, G. (M.) cf. lensiformis, G. (M.) formosa formosa, G. (Acarina) primitiva, G. (A.) cf. densa, G. (A.) cf. aspensis, G. (A.) broedermanni, Subbotina(?) taroubaensis, $S$. turgida, $S$. linaperta, S. inaequispira (group), G. (A.) soldadoensis angulosa, Pseudohastigerina micra.

Age: Zone P.8 (Subzone P.8b), Cuisian, Lower Eocene.
Sample 3-21A-3-1, 148-150 cm:

Neglecting the contamination from Pliocene to Recent horizons, the fauna of this sample is strongly heterogeneous with specimens from Zones P.8, P.7, P.6, P.5 and P.4 all represented. Accordingly it would seem that the sample represents mechanicalmixing of a condensed sequence representing the basal Eocene and later Paleocene.

Age: Zones P.8a to P.4 mixed.

Sample 3-21A-3-2, 148-150 cm:

As above.

Age: Zones P.8a to P.4 mixed.

Sample 3-21A-3-3, 148-150 cm:

As above.

Age: Zones P.8a to P.4 mixed.

Sample 3-21A-3-4, 148-150 cm:

As above.

Sample 3-21A-3-5, $87-89 \mathrm{~cm}$ :

As above. (Note: the bulk of the fauna seems referable to Zone P.4).

Age: As above, but possibly horizon is referable to Zone P.4.

Sample 3-21A-3-6, 74-76 cm:

Globorotalia (Morozovella) cf. velascoensis, G. (M.) acutispira, G. (M.) crosswickensis, G. (M.) cf. angulata, G. (Globorotalia) troelseni, G. (G.) pseudomenardii, G. (G.?) albeari, G. (Turborotalia) ehrenbergi, G. (T.) pusilla, Subbotina triloculinoides, Globorotalia (Acarinina) mckannai.

Age: Zone P.4 (earlier part), Thanetian, Paleocene.

Hole 22

Sample 3-22-1-1, 148-150 cm:

Rich planktonic foraminiferal fauna with Globorotalia (G.) truncatulinoides (s.l.), G. (G.) cultrata (s.l.), G. (G.) hirsuta hirsuta, G. (T.) inflata, Globigerina calida praecalida, G. falconensis, Globigerinoides conglobatus conglobatus, $G$. elongatus, etc.

Age: Zone N.22, Pleistocene.

Sample 3-22-1-2, 148-150 cm:

As above.

Sample 3-22-1-3, 148-150 cm:

As above.

Sample 3-22-1-4, 148-150 cm:

As above.

Sample 3-22-1-6, 63-65 cm:

As above.

Sample 3-22-1-1, 75-77 cm:

Globorotalia (T.) kugleri, G. (T.) pseudokugleri, G. (T.) 
peripheroronda forma $\propto, G$. (T.) cf. siakensis, G. (T.) cf. opima nana, Globigerinoides quadrilobatus immaturus, Globigerina sp. cf. falconensis, G. woodi woodi, $G$. angustiumbilicata.

Age: Zone N.4, basal Miocene, Lower Aquitanian.

Sample 3-22-2-2, 75-77 cm:

As above.

Sample 3-22-2-6, 73-75 cm:

As above, but with Globigerinoides quadrilobatus primordius.

Age: Zone N.4, basal Miocene.

Sample 3-22-3-1, 148-150 cm:

Globorotalia (T.) mendacis, G. (T.) cf. kugleri, G. (T.) cf. siakensis, G. (T.) cf. opima nana, G. (T.) permïcra, Cassigerinella chipolensis, Globigerina angulisuturalis, $G$. praebulloides occlusa, G. ouachitaensis ciperoensis, Globigerinita dissimilis (s.l.), G. unicava (s.l.).

Age: Zone N.3 (=P.22), Upper Oligocene.

Sample 3-22-3-2, 73-75 cm:

As above.

Sample 3-22-3-3, 148-150 cm:

As above.

Sample 3-22-3-4, 148-150 cm:

As above, but with Globoquadrina dehiscens praedehiscens.

Age: Zone N.3 (=P.22), upper Oligocene.

Sample 3-22-3-5, 148-150 cm:

Globigerina sellii, G. cf. galavisi, G. tripartita, G. angustiumbilicata, G. praebulloides (s.l.), Globorotalia (T.) opima opima, G. (T.) opima nana, G. (T.) permicra, G. (T.) mendacis, Globigerinita dissimilis (s.l.), G. unicava unicava, Globoquadrina dehiscens praedehiscens. Age: Zone N.2 (=P.21), upper Oligocene.

Sample 3-22-3-6, 74-76 cm:

As above.
Sample 3-22-4-1, 73-75 cm:

Globorotalia (T.) opima opima, G. (T.) opima nana, G. (T.) gemma, G. (T.) permicra, G. (T.) cf. siakensis, Globigerina angulisuturalis, G. anguliofficinalis, Globigerinita dissimilis (s.l.), G. unicava unicava, Globigerina prasaepis.

Age: Zone N.2 (=P.21), upper Miocene.

Sample 3-22-4-2, 81-83 cm:

G. (T.) opima opima, G. (T.) opima nana, G. (T.) gemma, G. (T.) permicra, Globigerina prasaepis, G. tripartita, $G$. cf. galavisi, $G$. anguliofficinalis, $G$. angustiumbilicata, G. cf. angulisuturalis, G. dissimilis (s.l.), G. unicava unicava, G. unicava cf. primitiva.

Age: ?Zone N.2 (=P.21), upper Miocene.

Sample 3-22-4-3, 148-150 cm:

Globigerina ampliapertura, G. anguliofficinalis, G. prasaepis, $G$. tripartita, $G$. cf. galavisi, G. (T.) opima opima, $G$. (T.) opima nana, G. (T.) gemma, G. (T.) permicra, Globigerinita unicava unicava, G. unicava cf. primitiva. Age: Zone N.1 (=P.20), upper Oligocene.

Sample 3-22-4-4, 77-79 cm:

As above.

Sample 3-22-4-5, 80-82 cm:

As above.

Sample 3-22-4-6, 78-80 cm:

As above.

Sample 3-22-5-1, 13-15 cm:

Acarinina densa, A. primitiva, Globorotalia (Morozovella) cf. "spinulosa", Subbotina linaperta, S. inaequispira (group), Globigerapsis kugleri, G. index, Globigerina (?) senni, Truncorotaloides rohri (group).

Age: Zone P.11/Zone P.12, Middle Eocene.

Sample 3-22-5-2, 140-142 cm:

As above.

\section{REFERENCE}

Blow, W. H., 1969. Late-Middle Eocene to Recent planktonic foraminiferal biostratigraphy. Proc 1st Inter. Conf. Plank. Microfossils. 1, 109. 